\title{
ON THE DECOMPOSITION OF TENSOR PRODUCTS OF PRINCIPAL SERIES REPRESENTATIONS FOR REAL-RANK ONE SEMISIMPLE GROUPS
}

\author{
BY \\ ROBERT PAUL MARTIN
}

\begin{abstract}
Let $G$ be a connected semisimple real-rank one Lie group with finite center. It is shown that the decomposition of the tensor product of two representations from the principal series of $G$ consists of two pieces, $T_{C}$ and $T_{d}$, where $T_{c}$ is a continuous direct sum with respect to Plancherel measure on $\hat{G}$ of representations from the principal series only, occurring with explicitly determined multiplicities, and $T_{d}$ is a discrete sum of representations from the discrete series of $G$, occurring with multiplicities which are, for the present, undetermined.
\end{abstract}

I. Introduction. Let $G$ be a connected semisimple Lie group with finite center. If $P$ is a cuspidal parabolic subgroup of $G$ and $P=M A N$ denotes a Langlands decomposition for $P$, we denote by $\hat{M}_{d}$ the square-integrable irreducible representations of $M$. For $\sigma \in \hat{M}_{d}, \tau \in \hat{A}, \sigma \times \tau$ is a representation of $M A N$ via $(\sigma \times \tau)($ man $)=\sigma(m) \tau(a)$ and the family of representations

$$
\left\{\pi(\sigma, \tau)=\operatorname{Ind}_{p}^{G} \sigma \times \tau: \sigma \in \hat{M}_{d}, \tau \in \hat{A}\right\}
$$

is called the nondegenerate continuous series corresponding to $\boldsymbol{P}$. In the case of a minimal parabolic subgroup, it is customary to say principal series. An important problem is that of decomposing the tensor product of two such representations into irreducibles. In $\S 5$, it is shown that this problem "reduces" to knowing how to decompose tensor products of representations from $\hat{M}_{d}$ and how to decompose $\operatorname{Ind}_{M A}^{G} \sigma \times \tau$ for all $(\sigma, \tau) \in \hat{M}_{d} \times \hat{A}$. One of the main goals of this paper is to show to what extent these last two problems can be answered when $G$ has real-rank one, $P=M A N$ is a minimal parabolic subgroup, and we are decomposing the tensor product of two principal series representations.

The main result is that this decomposition consists of two pieces, $T_{c}$ and

Presented to the Society, January 16, 1974 under the title Tensor products of principal series; received by the editors November 19, 1973.

AMS (MOS) subject classifications (1970). Primary 22E45; Secondary 22E43, 22D30.

Key words and phrases. Semisimple Lie groups, irreducible unitary representations, cuspidal parabolic subgroups, principal series, discrete series, tensor products. 
$T_{d}$, where $T_{c}$ is a continuous direct sum with respect to Plancherel measure on $\hat{G}$ of representations from the principal series only, occurring with explicitly determined multiplicities, and $T_{d}$ is a discrete direct sum of representations from the discrete series of $G$, occurring with multiplicities which are, for the present, undetermined. Let $V=\theta N$, where $\theta$ is an appropriate Cartan involution on $G$, and $S$ denote a suitably chosen cross-section for the action of $M A$ on $V$. Then both the cardinality of $S$ and the isotropy subgroup at $v_{0} \in S$ under the action of $M A$ (which is actually independent of $v_{0} \in S$ ) play decisive roles in determining the multiplicity of a principal series representation occuring in $T_{c}$.

For real-rank one groups we shall show, by using Mackey's tensor product theorem, that the problem of decomposing the tensor product of two principal series representations reduces to knowing $\operatorname{Ind}_{M A}^{G} \sigma \times \tau$ for all $\sigma \times \tau \in(M A)^{\wedge}$. We then show (in fact for arbitrary rank) that $\operatorname{Ind}_{M A}^{G} \sigma \times \tau$ is independent of $\tau \in \hat{A}$ and hence it suffices to determine $\operatorname{Ind}_{M A}^{G} \sigma \times \tau$ for almost all $\sigma \times \tau \in$ $(M A)^{\wedge}$. By applying a reciprocity theorem due to N. Anh, this amounts to determining the multiplicity of $\sigma \times \tau$ in the restriction of $\pi$ to $M A,(\pi)_{M A}$, for almost all $\pi \in \hat{G}$.

If $\pi$ is an irreducible representation from the principal series of $G$, then $(\pi)_{M A}$ is computed by applying Mackey's subgroup theorem to $M A N$ and $M A$. It is here that explicit knowledge of $V / M A$ is needed. If $S$ denotes a suitable cross-section for this action, then $S$ depends not only on the number of positive roots which are not simple roots but also on the dimensions of the root spaces.

For irreducible representations $\pi$ of the discrete series of $G$ we first give a new proof (one using Anh's reciprocity theorem) of the fact that there exists a $\delta \in \hat{K}$ such that $\pi$ is contained in $\operatorname{Ind}_{K}^{G} \delta$. We then use Mackey's subgroup theorem to compute $\left(\operatorname{Ind}_{K}^{G} \delta\right)_{M A}$. From this it becomes clear that

$$
(\pi)_{M A} \simeq \int_{M A}^{\oplus} n(\sigma, \tau, \pi)(\sigma \times \tau) d \mu_{C}(\sigma, \tau)
$$

where $\mu_{C}$ is Plancherel measure on $(M A)^{\wedge}$ and $n(\sigma, \tau, \pi) \in\{0,1,2, \cdots, \infty\}$.

The problem of decomposing the tensor product of principal series representations has been considered for SL $(2, \mathrm{C})$ by G. Mackey in [13] and M. A. Naimark in [14], for $\operatorname{SL}(n, C)$ by N. Anh in [1] , and was completely solved for complex semisimple groups by F. Williams in his 1972 thesis [18]. For $\operatorname{SL}(2, \mathbf{R})$, the problem was completely solved by L. Pukanszky in [15]. We shall comment on these cases in $\S 6$ of this paper and show how the techniques developed in $\S 5$ not only can be used to give new proofs of these results but also can be used to give a complete solution to the problem of decomposing the tensor product of two (minimal) principal series representations of $G=\mathrm{SL}(n, \mathbf{R}), n \geqslant 3$.

The author would like to thank Professor Ronald L. Lipsman for the many suggestions he made during the preparation of this manuscript. 
II. The semisimple theory. Let $G$ be a connected semisimple Lie group with finite center and Lie algebra $\mathfrak{g}$. Let $\mathfrak{g}=\mathfrak{t}+\mathfrak{p}$ be a Cartan decomposition, $\theta$ denote the corresponding Cartan involution, and $K=$ the maximal compact subgroup of $G$ with Lie algebra $\mathfrak{t}$. Let $B_{\theta}(X, Y)=-B(X, \theta Y)$ where $B$ is the Killing form and $X, Y \in g$. Then $B_{\theta}$ is an $\operatorname{Ad}(K)$-invariant inner product on $\mathfrak{g}$ which makes $\mathfrak{g}$ into a real Hilbert space. Let $\mathfrak{a}$ be a maximal (abelian) subalgebra of $\mathfrak{p}$ and $\mathfrak{a}^{*}=\operatorname{Hom}_{\mathbf{R}}(\boldsymbol{a}, \mathbf{R})$ its dual. For $\lambda \in \mathfrak{a}^{*}$, put $\mathfrak{g}_{\lambda}=$ $\{X \in \mathrm{g}:[H, X]=\lambda(H) X$, for all $H \in \mathfrak{a}\}$. If $\lambda \neq 0$ and $\boldsymbol{g}_{\lambda} \neq\{0\}$, then $\lambda$ is called a (restricted) root and $m_{\lambda}=\operatorname{dim} g_{\lambda}$ is called its multiplicity. For $\alpha \in \mathfrak{a}^{*}$, let $H_{\alpha} \in \mathfrak{a}$ be determined by $\alpha(H)=B_{\theta}\left(H, H_{\alpha}\right)$ for $H \in \mathfrak{a}$. Let $a^{\prime}$ be the open subset of $a$ where all restricted roots are $\neq 0$. The components of $\boldsymbol{a}^{\prime}$ are called Weyl chambers and if we fix a Weyl chamber $\mathfrak{a}^{+}$, a root $\alpha$ is called positive if it is positive on $a^{+}$. Let $\Delta$ (resp. $\Delta_{+}$) denote the set of roots (resp. positive roots). A root $\alpha \in \Delta_{+}$is called simple if it is not the sum of two positive roots. The simple roots form a basis for $a^{*}$. Put

$$
\mathfrak{n}=\sum_{\alpha \in \Delta_{+}} \mathfrak{g}_{\alpha}, \quad \mathfrak{v}=\theta \mathfrak{n}=\sum_{\alpha \in \Delta_{+}} \mathfrak{g}_{-\alpha},
$$

and let $N, V$ and $A$ denote the analytic subgroups of $G$ with Lie algebras $\mathfrak{n}, \mathfrak{b}$ and $a$ respectively. Then $G=K A N$ is an Iwasawa decomposition and the dimension of $A$ is called the real-rank of $G$. Let $M$ (resp. $M^{\prime}$ ) denote the centralizer (resp. normalizer) of $A$ in $K$. Then $M$ is normal in $M^{\prime}$, both $M, M^{\prime}$ are closed, $W=M^{\prime} / M$ is the (finite) Weyl group, and $M A N$ is a (minimal parabolic) subgroup of $G$.

If $m_{1}^{\prime}, \cdots, m_{w}^{\prime}$ is a set of representatives of $W$ and $P=M A N$, then we have the Bruhat decomposition $G=\bigcup_{i=1}^{w} P m_{i}^{\prime} P$. This union is disjoint and exactly one of the summands, viz., $P m^{*} P$ where $\operatorname{Ad}\left(m^{*}\right) \mathfrak{a}^{+}=\mathfrak{a}^{-}=$ $\{H: \alpha(H)<0$, for all $\alpha>0\}$, is open in $G$. So $M A N V$ has a complement of measure zero in $G$.

By a parabolic subgroup $P$ of $G$ is meant a closed subgroup of $G$ such that

(i) if $\mathfrak{b}=L A(P)$, then $P$ is the normalizer of $\mathfrak{b}$ in $G$, and

(ii) $\mathfrak{b}_{\mathbf{c}}$ contains a maximal solvable subalgebra of $\boldsymbol{g}_{\mathrm{C}}$. A parabolic subgroup $\boldsymbol{P}$ is called minimal if it is minimal among all parabolic subgroups of $G$. Let $N=$ the maximal unipotent normal subgroup of $P$, set $\Xi=P \cap \theta P$, and set $A=$ the maximal connected split (i.e., $\operatorname{Ad}(a)$ diagonizable over R) abelian subgroup lying in the center of $\Xi$. Then $\Xi$ is the centralizer of $A$ in $G$. Let $X(\Xi)=\left\{\chi: \Xi \rightarrow \mathbf{R}^{*}, \chi\right.$ a continuous homomorphism $\}$. Set $M=\bigcap_{\chi \in X(\Xi)}$ ker $|\chi|$. Then $M$ is reductive (i.e., $\mathfrak{m}=L A(M)$ is reductive) but not connected in general. Moreover, $\Xi=M A$ is a direct product and 
the map $(m, a, n) \rightarrow$ man is an analytic diffeomorphism of $M \times A \times N$ onto $P$. This is called the Langlands decomposition for $P$. $P$ is said to be cuspidal if $M$ has a compact Cartan subgroup. For the results quoted above, we refer to [5] and [17].

Let $P=M A N$ be a cuspidal parabolic subgroup of $G$ and $\hat{M}_{d}$ denote the square-integrable irreducible representations of $M$. Then for $\tau \in \hat{A}, \sigma \in \hat{M}_{d}$, $\sigma \times \tau$ is a representation of $M A N$ via $(\sigma \times \tau)($ man $)=\sigma(m) \tau(a)$ and the family of representations $\left\{\pi(\sigma, \tau)=\operatorname{Ind}_{P}^{G} \sigma \times \tau: \sigma \in \hat{M}_{d}, \tau \in \hat{A}\right\}$ is called the nondegenerate continuous series corresponding to $P$. In the case of a minimal parabolic subgroup, it is customary to say principal series. It is known that almost all the $\pi(\sigma, \tau)$ are irreducible and, as in the case of a minimal parabolic subgroup of $G, \operatorname{MAN} \bar{N}(\bar{N}=\theta N)$ has a complement of measure zero in $G$. Two cuspidal parabolics $P_{1}, P_{2}$ are called associate if there exists $x \in G$ such that $x A_{1} x^{-1}$ $=A_{2}$. Conjugating by $x$, we may assume that $P_{1}=M A N_{1}, P_{2}=M A N_{2}$. Finally, it can be shown that $\operatorname{Ind}_{P_{1}}^{G} \sigma \times \tau \simeq \operatorname{Ind}_{P_{2}}^{G} \sigma \times \tau$, for $\sigma \in \hat{M}_{d}, \tau \in \hat{A}$ and $P_{1}=M A N_{1}, P_{2}=M A N_{2}$ associate parabolics [11, p. 473].

III. Results of Mackey and Anh. Let $X$ be a locally compact Hausdorff space, $\mu$ a finite Borel measure on $X, R$ an equivalence relation on $X, Y=$ $X / R$, and $r: X \rightarrow Y$ the canonical projection. Then $R$ is said to be a measurable equivalence relation if there exists a countable collection $\left\{E_{i}\right\}$ of subsets of $Y$ such that $r^{-1}\left(E_{i}\right)$ is measurable and for every $y \in Y,\{y\}=$ $\bigcap\left\{E_{i}: y \in E_{i}\right\}$. Now let $G_{1}, G_{2}$ be closed subgroups of the separable locally compact group $G . G_{1}, G_{2}$ are said to be regularly related if there exist measurable sets $E_{0}, E_{1}, \cdots$ in $G$ such that each $E_{i}$ is a union of $G_{1}: G_{2}$ double cosets, $E_{0}$ has measure zero, and every double coset outside of $E_{0}$ is the intersection of the $E_{i}$ that contain it. Let $D=$ the collection of $G_{1}: G_{2}$ double cosets in $G$. Clearly $G_{1}: G_{2}$ are regularly related if and only if the double cosets outside of a certain set of measure zero form the equivalence classes of a measurable equivalence relation. If $\mu$ is a finite measure on $G$ equivalent to Haar measure and $r: G \rightarrow D$ is the projection, then the measure $\nu$ given by $\nu(E)=\nu\left(r^{-1}(E)\right)$, whenever $E$ is such that $r^{-1}(E)$ is measurable, is called an admissible measure on $D$. Any two such are equivalent. In the special case where there exists a subset of $G$ with complement of measure zero which is itself the countable union of $G_{1}: G_{2}$ double cosets, then $G_{1}: G_{2}$ are called discretely related. In this case $\nu$ is a discrete measure.

THEOREM (MACKeY'S SUBGROUP THEOREM [12, p. 127]). Let $G$ be a separable locally compact group, $G_{1}, G_{2}$ be regularly related closed subgroups of $G$, and $\pi \in \operatorname{Rep}\left(G_{1}\right)$. For each $x \in G$ consider $G_{x}=G_{2} \cap x^{-1} G_{1} x$. Form $V_{x}=$ $\operatorname{Ind}_{G_{x}}^{G_{2}}\left(\eta \rightarrow \pi\left(x \eta x^{-1}\right)\right), \eta \in G_{x}$ Then $V_{x}$ is determined to within equivalence by the $G_{1}: G_{2}$ double coset $d$ to which $x$ belongs, write it $V_{d}$, and 


$$
\left(\operatorname{Ind}_{G_{1}}^{G} \pi\right)_{G_{2}} \simeq \int_{D}^{\oplus} V_{d} d \nu(d)
$$

where $\nu$ is any admissible measure on $D=G_{1} \backslash G / G_{2}$.

TheOREM (MACKey'S tensor PRoduct theorem [12, p. 128]). Let $G$, $G_{1}, G_{2}$ be as in Mackey's subgroup theorem, $\pi_{1} \in \operatorname{Rep}\left(G_{1}\right)$, and $\pi_{2} \in$ $\operatorname{Rep}\left(G_{2}\right)$. For $(x, y) \in G \times G$ denote

$$
\begin{gathered}
G_{x, y}=x^{-1} G_{1} x \cap y^{-1} G_{2} y, \\
\pi_{x, y}(g)=\pi_{1}\left(x g x^{-1}\right) \otimes \pi_{2}\left(y g y^{-1}\right), \text { and } \pi^{x, y}=\operatorname{Ind}_{G_{x, y}}^{G} \pi_{x, y} .
\end{gathered}
$$

Then $\pi^{x, y}$ is determined to within equivalence by the double coset $d$ to which $x y^{-1}$ belongs, write it $\pi^{d}$, and if $\nu$ is any admissible measure on $D=$ $G_{1} \backslash G / G_{2}$, then

$$
\operatorname{Ind}_{G_{1}}^{G} \pi_{1} \otimes \operatorname{Ind}_{G_{2}}^{G} \pi_{2} \simeq \int_{D}^{\oplus} \pi^{d} d \nu(d)
$$

The following generalization of Mackey's reciprocity theorem [13, p. 212] will play an important role in $\$ 5$.

THEOREM (ANH [1, p. 299]). Let $G$ by a type I separable locally compact group, $H \subseteq G$ a closed type I subgroup, $\mu_{G}, \mu_{H}$ finite measures in the Plancherel measure classes of $G, H$ respectively, $\omega(\pi, \nu)$ and $n(\pi, \nu)$ be $\mu_{G} \times \mu_{H}$ measurable functions where $n(\pi, \nu)$ is a countable cardinal for every $\pi, \nu$. Then the following are equivalent:

(i) For $\mu_{H}$-aimost all $\nu$,

$$
\operatorname{Ind}_{H}^{G} \nu \simeq \int_{\hat{G}}^{\oplus} n(\pi, \nu) \pi \omega(\pi, \nu) d \mu_{G}(\pi) .
$$

(ii) For $\mu_{G}$-almost all $\pi$,

$$
(\pi)_{H} \simeq \int_{\hat{H}}^{\oplus} n(\pi, \nu) \nu \omega(\pi, \nu) d \mu_{H}(v) .
$$

IV. Real-rank one Lie groups and algebras. Let $G$ be a real-rank one connected semisimple Lie group with finite center, Lie algebra $\mathfrak{g}$, and Iwasawa decomposition $K A N$. Then $\operatorname{dim} A=1$ and, if $\alpha$ denotes a simple (restricted) root of $8, \alpha$ may be chosen so that all roots are of the form $j \alpha, j= \pm 1, \pm 2$. Let $V=\theta N, M=$ the centralizer of $A$ in $K, M^{\prime}=$ the normalizer of $A$ in $K, P=M A N$, and $W$ the finite (Weyl) group $M^{\prime} / M$. $W$ has order two and if $m^{\prime} \in M^{\prime}-M$, we have the Bruhat decomposition $G=M A N \cup M A N m^{\prime} M A N$ (and so there are only two $M A N: M A N$ double cosets in $G$ with only one of positive measure and $M A N V$ has a complement of Haar measure zero in $G$ ). If $\mathfrak{a}, \mathfrak{m}$ denote the Lie algebras of $A, M$ and $\mathfrak{g}_{j \alpha}=\{X \in \mathfrak{g}:[H, X]=$ $j \alpha(H) X$, all $H \in a\}$, then 


$$
\mathfrak{g}=\mathfrak{m} \oplus \mathfrak{a} \oplus \mathfrak{g}_{\alpha} \oplus \mathfrak{g}_{2 \alpha} \oplus \mathfrak{g}_{-\alpha} \oplus \mathfrak{g}_{-2 \alpha} .
$$

If $\boldsymbol{g}$ is simple, then $\mathfrak{g}$ is $\operatorname{so}(n, 1), \operatorname{su}(n, 1), \operatorname{sp}(n, 1)$ or $\mathrm{f}_{4,9}$ (i.e., $G$ is locally isomorphic to $\mathrm{SO}_{e}(n, 1), \mathrm{SU}(n, 1), \mathrm{SP}(n, 1)$, or $\left.\mathrm{F}_{4,9}\right)$. If 8 is semisimple, then

$$
\mathfrak{g}=\sum_{i=1}^{r} \mathfrak{g}_{i}=\sum_{1}^{r}\left(\mathfrak{f}_{i}+a_{i}+\mathfrak{n}_{i}\right)
$$

where each $\mathfrak{g}_{i}$ is a simple ideal $\left[5\right.$, p. 122] (and so each $\boldsymbol{g}_{i}$ centralizes $\boldsymbol{g}_{j}$, $i \neq j$ ) and $\mathfrak{l}_{\boldsymbol{i}}+\mathfrak{a}_{\boldsymbol{i}}+\mathfrak{n}_{\boldsymbol{i}}$ denotes an Iwasawa decomposition for $\boldsymbol{g}_{\boldsymbol{i}}$. Since $\mathfrak{g}$ has real-rank one, all but exactly one of the $\mathfrak{g}_{i}$, say $g_{r}$, must be compact and so $\mathfrak{g}=\left(\mathfrak{l}_{1}+\mathfrak{l}_{2}+\cdots+\mathfrak{l}_{r}\right)+\mathfrak{a}_{r}+\mathfrak{n}_{r}$ will be an Iwasawa decomposition for $g$. Let

$$
\widetilde{K}=\left(\exp \mathfrak{t}_{1}\right)\left(\exp \mathfrak{t}_{2}\right) \cdots\left(\exp t_{r-1}\right)
$$

Then

$$
\begin{aligned}
& G=\left(\exp \mathfrak{\ell}_{1}\right)\left(\exp \mathfrak{f}_{2}\right) \cdots\left(\exp \mathfrak{f}_{r}\right) A_{r} N_{r}=\widetilde{K} K_{r} A_{r} N_{r}, \\
& K=\widetilde{K} K_{r}, \quad A=A_{r}, \quad N=N_{r}, \quad M=\widetilde{K} M_{r} .
\end{aligned}
$$

Note that since $\operatorname{Ad}(\widetilde{K})$ fixes $\mathfrak{n}=L A(N)$ (or $\mathfrak{v}=\theta \mathfrak{n}$ ), in determining the orbits of points in $\mathfrak{n}$ (or $\mathfrak{b}$ ) under the action of $M A$, we may assume that $G$ is simple (of course the stability groups will in general be larger). Let us examine the simple real-rank one groups more closely.

A. The classical cases. We shall look more closely at $\mathrm{SO}_{e}(n, 1), \mathrm{SU}(n, 1)$, $\operatorname{SP}(n, 1)$ for $n \geqslant 2$ and $\operatorname{Spin}(n, 1)$ for $n \geqslant 3$ (recall that $\operatorname{SU}(1,1)$ is locally isomorphic to $\mathrm{SO}_{e}(2,1), \mathrm{SP}(1,1)$ is locally isomorphic to $\mathrm{SO}_{e}(4,1)$ [5, p. 351], and $\mathrm{SO}_{e}(1,1) \approx \mathbf{R}^{x}$ is not semisimple).

Let $n \geqslant 2$ and $\mathbf{K}$ be $\mathbf{R}, \mathbf{C}$ or $H$ (the quaternions). Let $G$ be the group of all automorphisms of $\mathrm{K}^{n+1}$ which preserve the hermitian quadratic form $\left|x_{1}\right|^{2}+\cdots+\left|x_{n}\right|^{2}-\left|x_{n+1}\right|^{2}$ with the additional property that if $\mathbf{K}=\mathbf{R}$ or $\mathbf{C}$, we consider only automorphisms of determinant 1 . Then $G$ is $\mathrm{SO}(n, 1), \mathrm{SU}(n, 1)$ or $\mathrm{SP}(n, 1)$ according to whether $\mathbf{K}$ is $\mathbf{R}, \mathbf{C}$ or $H[8$, p. 555]. $\mathrm{SU}(n, 1)$ and $\mathrm{SP}(n, 1)$ are connected $[5$, p. 346] and we denote by $\mathrm{SO}_{e}(n, 1)$ the identity component of $\mathrm{SO}(n, 1)$. Then we have a Cartan decomposition $\mathfrak{g}=\mathfrak{l}+\mathfrak{p}$ for the Lie algebra of $G$ where:

$$
\begin{aligned}
\mathfrak{l}=\left(\begin{array}{cc}
X_{1} & 0 \\
0 & X_{2}
\end{array}\right), & X_{1} \text { is an } n \times n \text { skew hermitian with } \\
& \text { entries in } \mathrm{K}, X_{2} \text { is a skew member of } \\
& \mathrm{K}, X_{2}+\operatorname{tr} X_{1}=0 \text { if } \mathrm{K}=\mathrm{C}\left(X_{2}=0\right. \\
& \text { for } \mathrm{K}=\mathrm{R}),
\end{aligned}
$$




$$
\mathfrak{p}=\left(\begin{array}{cc}
0 & Y \\
\bar{Y}^{t} & 0
\end{array}\right), \quad Y \text { is a column vector in } \mathbf{K}^{n} .
$$

In each case the Cartan involution, $\theta$, is negative conjugate transpose. We also have:

$$
\begin{aligned}
& a=R \cdot\left(\begin{array}{ll}
0 & 1 \\
1 & 0
\end{array}\right), \quad A=\left(\begin{array}{ccc}
\cosh t & 0 & \sinh t \\
0 & I & 0 \\
\sinh t & 0 & \cosh t
\end{array}\right), \\
& g_{\alpha}=\left(\begin{array}{ccc}
0 & \bar{X}^{t} & 0 \\
-X & 0 & X \\
0 & \bar{X}^{t} & 0
\end{array}\right), \quad X \text { a column vector in } \mathbf{K}^{n-1}, \\
& \boldsymbol{g}_{2 \alpha}=\left(\begin{array}{ccc}
Y & 0 & -Y \\
0 & 0 & 0 \\
Y & 0 & -Y
\end{array}\right), \quad \begin{array}{l}
\mathfrak{g}_{2 \alpha}=0 \text { if } \mathrm{K}=\mathrm{R} ; Y \in \mathrm{K} \text { with } \\
\bar{Y}=-Y \text { if } \mathrm{K}=\mathrm{C} \text { or } H,
\end{array} \\
& \mathfrak{n}=\mathfrak{g}_{\alpha} \oplus \mathfrak{g}_{2 \alpha}, \quad \mathfrak{b}=\theta \mathfrak{n}, \\
& V=\left(\begin{array}{ccc}
1+Y-1 / 2 \mid X R^{2} & \bar{X}^{t} & Y-1 / 2|X|^{2} \\
-X & I & -X \\
-Y+1 / 2|X|^{2} & -\bar{X}^{t} & 1-Y+1 / 2|X|^{2}
\end{array}\right) \\
& N=\left(\begin{array}{ccc}
1+Y-1 / 2|X|^{2} & \bar{X}^{t} & -Y+1 / 2|X|^{2} \\
-X & I & X \\
Y-1 / 2|X|^{2} & \bar{X}^{t} & 1-Y+1 / 2|X|^{2}
\end{array}\right), \\
& K= \begin{cases}\left(\begin{array}{ll}
k & 0 \\
0 & 1
\end{array}\right) \approx \mathrm{SO}(n), \quad k \in \mathrm{SO}(n) & \text { for } \mathbf{K}=\mathbf{R}, \\
\left(\begin{array}{ll}
u & 0 \\
0 & c
\end{array}\right) \approx S(U(n) \times U(1)) \approx U(n), & u \in U(n), \quad|c|=1, \\
& c \operatorname{det} u=1 \text { for } \mathbf{K}=\mathbf{C}, \\
\left(\begin{array}{ll}
\omega & 0 \\
0 & u
\end{array}\right) \approx \mathrm{Sp}(n) \times \mathrm{Sp}(1), & \begin{array}{l}
\omega \in \mathrm{Sp}(n), \quad u \in \mathrm{Sp}(1) \\
\text { (unit quaternions) for } \mathbf{K}=H,
\end{array}\end{cases} \\
& \mathfrak{m}=\left(\begin{array}{lll}
X_{2} & 0 & 0 \\
0 & X & 0 \\
0 & 0 & X_{2}
\end{array}\right), \begin{array}{l}
X_{2} \text { skew in } \mathrm{K}(=0 \text { if } \mathrm{K}=\mathrm{R}) \\
X \text { an }(n-1) \times(n-1) \text { skew hermitian }
\end{array} \\
& \text { with entries in } \mathrm{K}, 2 X_{2}+\operatorname{tr} X=0 \\
& \text { if } \mathbf{K}=\mathbf{C} \text {, }
\end{aligned}
$$




$$
M=\left\{\begin{array}{cl}
\left(\begin{array}{ccc}
1 & 0 & 0 \\
0 & u & 0 \\
0 & 0 & 1
\end{array}\right), & u \in \operatorname{SO}(n-1) \text { for } \mathbf{K}=\mathbf{R}, \\
\left(\begin{array}{lll}
c & 0 & 0 \\
0 & \lambda & 0 \\
0 & 0 & c
\end{array}\right), & |c|=1, \lambda \in U(n-1), c^{2} \operatorname{det} \lambda=1 . \\
\left(\begin{array}{lll}
u & 0 & 0 \\
0 & \omega & 0 \\
0 & 0 & u
\end{array}\right), & u \in \operatorname{Spr}(1), \omega \in \operatorname{Sp}(n-1) \text { for } \mathbf{K}=H .
\end{array}\right.
$$

To investigate the action of $M A$ on $V$, we deal with an algebra conjugate to 8. Let

$$
s_{0}=\left(\begin{array}{lll}
2^{-1 / 2} & 0 & 2^{-1 / 2} \\
0 & I & 0 \\
-2^{-1 / 2} & 0 & 2^{-1 / 2}
\end{array}\right)
$$

and $8=s_{0} g s_{0}^{-1}$. Then $A$ becomes

$$
\left(\begin{array}{lll}
e^{t} & 0 & 0 \\
0 & I & 0 \\
0 & 0 & e^{-t}
\end{array}\right)
$$

$M$ remains the same,

and

$$
N=\left(\begin{array}{ccc}
1 & \bar{X}^{t} & 1 / 2\left(Y+|X|^{2}\right) \\
0 & I & X \\
0 & 0 & 1
\end{array}\right),
$$

The action of $A$ on $V$ is:

$$
V=\exp (X+Y) \equiv(X, Y)=\left(\begin{array}{ccc}
1 & 0 & 0 \\
X & I & 0 \\
1 / 2\left(Y+|X|^{2}\right) & \bar{X}^{t} & 1
\end{array}\right)
$$

$$
\begin{aligned}
\left(\begin{array}{lll}
e^{t} & 0 & 0 \\
0 & I & 0 \\
0 & 0 & e^{-t}
\end{array}\right)\left(\begin{array}{ccc}
1 & 0 & 0 \\
X & I & 0 \\
1 / 2\left(Y+|X|^{2}\right) & \bar{X}^{t} & 1
\end{array}\right)\left(\begin{array}{ccc}
e^{-t} & 0 & 0 \\
0 & I & 0 \\
0 & 0 & e^{t}
\end{array}\right) \\
=\left(\begin{array}{ccc}
1 & 0 & 0 \\
e^{-t} X & I & 0 \\
e^{-2 t_{1 / 2}\left(Y+|X|^{2}\right)} & e^{-t} \bar{X}^{t} & 1
\end{array}\right)
\end{aligned}
$$


i.e., $a \cdot v=e^{t} \cdot(X, Y)=\left(e^{-t} X, e^{-2 t} Y\right)$ while the action of $M$ on $V$ is:

$$
\begin{aligned}
& \left(\begin{array}{lll}
u & 0 & 0 \\
0 & \omega & 0 \\
0 & 0 & u
\end{array}\right)\left(\begin{array}{ccc}
1 & 0 & 0 \\
X & I & 0 \\
1 / 2\left(Y+|X|^{2}\right) & \bar{X}^{t} & 1
\end{array}\right)\left(\begin{array}{lll}
u^{-1} & 0 & 0 \\
0 & \cdots)^{-1} & 0 \\
0 & 0 & u^{-1}
\end{array}\right) \\
& \quad=\left(\begin{array}{ccc}
1 & 0 & 0 \\
\omega X u^{-1} & I & 0 \\
u^{1 / 2}\left(Y+|X|^{2}\right) u^{-1} & u \bar{X}^{t} \omega^{-1} & 1
\end{array}\right)=\left(\begin{array}{ccc}
1 & 0 & 0 \\
\omega X u^{-1} & I & 0 \\
1 / 2\left(u Y u^{-1}+|X|^{2}\right) & u \bar{X}^{t} \omega^{-1} & 1
\end{array}\right)
\end{aligned}
$$

i.e., $m \cdot v=\operatorname{diag}(u, \omega, u) \cdot(X, Y)=\left(\omega X u^{-1}, u Y u^{-1}\right)$. In each case the action of $M$ is by rotation while the action of $A$ is by dilation. For $\mathrm{K}=\mathrm{R}, M A$ acts on $V^{x}=V-\{0\}$ in one orbit for $n \geqslant 3$ and two orbits for $n=2$. For $\mathrm{K}=\mathrm{C}, M A$ acts on $\exp g_{-\alpha}$ by rotation and dilation by $e^{-t}$ while only $A$ acts on $\exp g_{-2 \alpha}\left(M\right.$ fixes $\exp g_{-2 \alpha}$ ) by dilation by $e^{-2 t}$. So $M A$ acts transitively on $\exp g_{-\alpha}^{x}$ while $M A$ acts on $\exp 8_{-2 \alpha}^{x}$ in two orbits. If we take $X_{-\alpha} \in 8_{-\alpha}^{x}=g_{-\alpha}-\{0\}, Y_{-2 \alpha} \in 8_{-2 \alpha}^{x}$, then up to a set of Haar measure zero in $V,\left\{\exp \left(t X_{-\alpha} \pm Y_{-2 \alpha}\right): t>0\right\}$ will serve as a cross-section for the action of $M A$ on $V=\exp \left(g_{-\alpha} \oplus g_{-2 \alpha}\right)$. For $\mathrm{K}=H, M=\operatorname{Sp}(1)$ $\times \mathrm{Sp}(n-1)$ where both $\mathrm{Sp}(1)$ and $\mathrm{Sp}(n-1)$ act on $\exp 8_{-\alpha}$ while only $\operatorname{Sp}(1)$ acts on $\exp g_{-2 \alpha}$. One may easily verify that the above action of $\mathrm{Sp}$ (1) on $\exp 8_{-2 \alpha}$ is the same as that of SO (3) on $\mathbf{R}^{3}[2, \mathrm{p} .38]$. In this case $M A$ will act transitively on both $\exp 8_{-\alpha}^{x}$ and $\exp 8_{-2 \alpha}^{x}$, in fact if $M_{Y}=$ $\left\{m \in M: m \cdot \exp Y=\exp Y, Y \in \boldsymbol{g}_{-2 \alpha}^{x}\right\}$, then $M_{Y} A$ also acts transitively on $\exp \boldsymbol{g}_{-\alpha}^{x}$. So if we take $X_{-\alpha} \in \boldsymbol{g}_{-\alpha}^{x}, Y_{-2 \alpha} \in \boldsymbol{g}_{-2 \alpha}^{x}$, then up to a set of Haar measure zero in $V,\left\{\exp \left(t X_{-\alpha}+Y_{-2 \alpha}\right): t>0\right\}$ will be a cross-section for the action of $M A$ on $V$.

For $n \geqslant 3, \operatorname{Spin}(n, 1)$ denotes the simply-connected double covering group of $\mathrm{SO}_{e}(n, 1)$. (Recall that the universal covering of $\operatorname{SL}(2, \mathbf{R})$ or $\mathrm{SU}(n, 1)$ does not have a finite center and that $\operatorname{Sp}(n, 1)$, as well as $\mathrm{F}_{4,9}$, is already simply connected.) If $\widetilde{G}=\operatorname{Spin}(n, 1)=\widetilde{K} \widetilde{A} \widetilde{N}$ and $G=\operatorname{SO}_{e}(n, 1)=$ $K A N$, then we have $\widetilde{K}=\operatorname{Spin}(n), \widetilde{K} / Z_{2} \approx K, \widetilde{A}=A, \widetilde{N}=N, \widetilde{M}=$ $\operatorname{Spin}(n-1)$, and $\widetilde{M} / Z_{2} \approx M$. Clearly $\widetilde{M} A$ acts transitively on $V^{x}$.

B. The exceptional case: $\mathrm{F}_{4,9}$. There is only one nonclassical simple Lie group of real-rank one. It is a real form of $F_{4}[5$, p. 354]. Denote this group by $F_{4,9}$ and its Lie algebra by $f_{4,9}$. Then $\operatorname{dim} f_{4,9}=52, \operatorname{dim} g_{2 \alpha}=7$, $\operatorname{dim} \boldsymbol{g}_{\alpha}=8, K=\operatorname{Spin}(9)$, and $M=\operatorname{Spin}$ (7) (see [6]).

C. The only remaining simple groups of real-rank one are quotients of the universal covering groups of the above groups by discrete central subgroups $Z$ 
$(\subseteq M)$. Note that if $G=K A N$ is such a universal covering group, $Z$ is a discrete central subgroup of $G$, and $G^{\prime}=G / Z=K^{\prime} A^{\prime} N^{\prime}$, then

$$
K^{\prime}=K / Z, A^{\prime}=A, N^{\prime}=N \text {, and } M^{\prime}=M / Z \text {. }
$$

In particular for $m^{\prime}=m Z \in M, X \in \mathfrak{n}$ (or $\left.b\right), \operatorname{Ad}\left(m^{\prime}\right) X=\operatorname{Ad}(m Z) X=\operatorname{Ad}(m) X$ and so in computing the orbits of points $n$ (or $b$ ) under the action of $M^{\prime} A^{\prime}$, we may assume that $G^{\prime}$ or $G$ is one of the groups described in $A$ or $B$, i.e., $\mathrm{SO}_{e}(n, 1), \mathrm{SU}(n, 1), \mathrm{Sp}(n, 1)$, or $\mathrm{F}_{4,9}$.

Let us remark at this time that since $M A$ can be regarded as a direct product, the irreducible representations $L \in(M A)^{\wedge}$ are all of the form $L=$ $\sigma \times \tau$ with $\sigma \in \hat{M}, \tau \in \hat{A}$ and so $(M A)^{\wedge}=\hat{M} \times \hat{A}$ with Plancherel measure on $(M A)^{\wedge}$ being the product of the Plancherel measures on $\hat{M}$ and $\hat{A}$ [7]. We shall put $M A=C$ where convenient and shall denote by $\mu_{C}$ (resp. $\mu_{G}$ ) Plancherel measure on $\hat{C}$ (resp. $\hat{G}$ ). We now thank Anthony Knapp for supplying the details of the following argument which is due to Kostant and lies behind some of this work in [9].

Lemma. Let 8 be a real-rank one semisimple Lie algebra and $k= \pm 1$, \pm 2 . Then

(i) if $\operatorname{dim} 8_{k \alpha}=1$, then $M A$ will act on $8_{k \alpha}-\{0\}=8_{k \alpha}^{x}$ in two orbits while

(ii) if $\operatorname{dim} g_{k \alpha}>1$, then $M A$ will act on $\boldsymbol{g}_{k \alpha}^{x}$ in one orbit.

Proof. As stated earlier in this section, we may assume $g$ is simple. Note also that the above lemma follows immediately from previous calculations when $g$ is one of the classical real-rank one algebras. The following general argument shows the result to be true for $f_{4,9}$ as well. As noted above, we may assume $\operatorname{dim} 8_{k \alpha}>1$ for $k= \pm 1, \pm 2$.

Let $k=1, X \in \mathbb{8}_{\alpha}$, and $H_{\alpha} \in \mathfrak{a}$ be determined by $\alpha(H)=B_{\theta}\left(H, H_{\alpha}\right)$, $H \in a$. Then $\theta X \in 8_{-\alpha},[X, \theta X]=B(X, \theta X) H_{\alpha} \in \mathfrak{a}$, and $\left\{X, \theta X, H_{\alpha}\right\}$ spans a subalgebra of 8 isomorphic to $\mathrm{sl}(2, \mathrm{R})$. Left multiplication by this subalgebra gives a representation of $\mathrm{sl}(2, \mathrm{R})$ on 8 and $\left\{X, \theta X, H_{\alpha}\right\}$ spans an invariant subspace. Any finite-dimensional representation of $\mathrm{sl}(2, R)$ splits into a direct sum of irreducibles and we may assume that these irreducibles are orthogonal with respect to the natural inner product on $\mathbf{8}$. One of these irreducibles is $\left\{X, \theta X, H_{\alpha}\right\}$ with weights (i.e., the eigenvalues of ad $H_{\alpha}$ ) $\alpha, 0,-\alpha$. Matching these weights with the weights of all the irreducible abstract representations of $\operatorname{sl}(2, \mathbf{R})$, we see that the weights of an $n$-dimensional representation would have to be $1 / 2(n-1) \alpha, 1 / 2(n-3) \alpha, \cdots,-1 / 2(n-1) \alpha$. The only possible weights in $g$ are $2 \alpha, \alpha, 0,-\alpha,-2 \alpha$ and so $n=1,3$ or 5 . We now have the following diagram: 


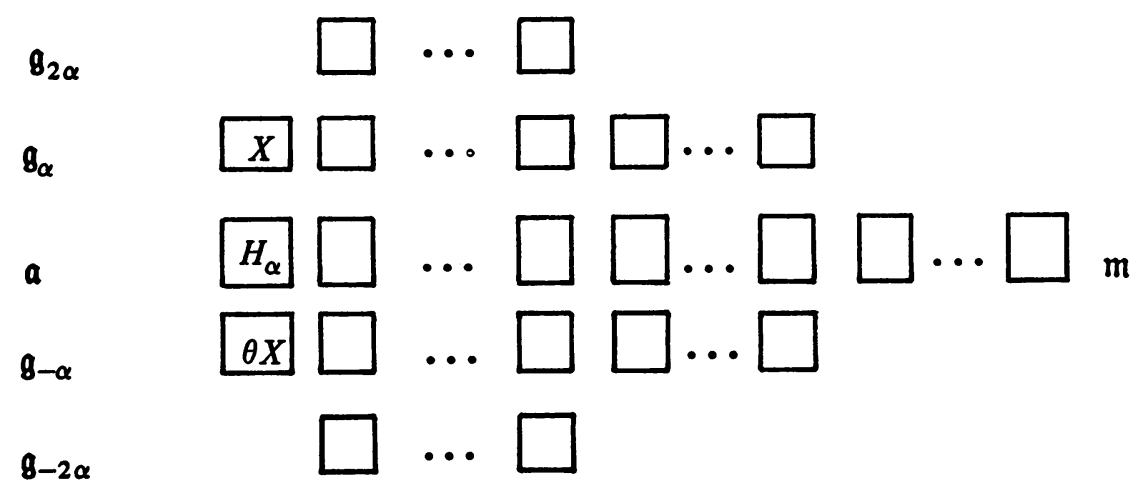

where each vertical column represents an invariant subspace and each box represents a one-dimensional subspace. Note that ad $X$ of each box in the $a+m$ row is contained in the corresponding box in the $8_{\alpha}$ row. Let $X=X_{1}, \cdots$, $X_{p}$ be a basis for the $8_{\alpha}$ row and choose basis elements $H_{1}, \cdots, H_{p}, H_{p+1}$, $\cdots, H_{q}$ in the $a+m$ row such that $\left[X, H_{i}\right]=X_{i}, i=1, \cdots, p$ (e.g., $\left.H_{1}=-H_{\alpha} / \alpha\left(H_{\alpha}\right)\right)$. Any vector $Y \in \mathbb{B}_{\alpha}$ is a linear combination of the $X_{i}$ 's, say $Y=\sum_{i=1}^{p} c_{i} X_{i}$. Let $W=\Sigma_{i=1}^{p} c_{i} H_{i} \in a+\mathfrak{m}$. Then

$$
[X, W]=\sum_{i=1}^{p} c_{i}\left[X, H_{i}\right]=\sum_{i=1}^{p} c_{i} X_{i}=Y
$$

and we have shown that $[X, a+m]=g_{\alpha}$ for $X \in g_{\alpha}-\{0\}$.

Now we consider the map $f: M A \rightarrow g_{\alpha}$ via $\operatorname{ma} \mapsto \operatorname{Ad}(m a) X$. Since $d f: a+m \rightarrow g_{\alpha}$ is given by $m+a \mapsto \operatorname{ad}(m+a) X=[m+a, X]$, the above argument shows that $d f$ is onto and so it follows from the inverse function theorem that the orbit $\operatorname{Ad}(M A) X$ is open in $g_{\alpha}-\{0\}$. Since $M$ is compact and the action of $A$ is given by

$$
a \cdot X=\operatorname{Ad}(a) X=e^{\operatorname{ad}(H)} X=e^{\alpha(H)} X, \quad a=\exp H, H \in \mathfrak{a},
$$

it follows that this orbit is also closed in $g_{\alpha}-\{0\}$. Since $\operatorname{dim} g_{\alpha}>1, \operatorname{Ad}(M A)$ acts in $8_{\alpha}-\{0\}$ in one orbit.

The proofs for $k=-1, \pm 2$ are similar and the lemma has been proven.

Note. Let $c \in M A, X \in \mathrm{g}_{k \alpha}$. Since $c \cdot \exp X=c(\exp X) c^{-1}=$ $\exp \operatorname{Ad}(c) X=\exp (c \cdot X)$, there is a canonical 1-1 correspondence between orbits in $g_{k \alpha} / M A$ and orbits in $\left(\exp g_{k \alpha}\right) / M A$. Thus $M A$ will act on $\exp \left(g_{k \alpha}^{x}\right)$ in one orbit for $\operatorname{dim} g_{k \alpha}>1$ and two orbits for $\operatorname{dim} g_{k \alpha}=1$.

V. The tensor product of principal series representations. Let $G$ be a connected semisimple Lie group with finite center, $P_{1}=M A N$ be a cuspidal parabolic subgroup of $G$, and $P_{2}=M A \bar{N}$ where $\bar{N}=\theta N$. Recall that $\hat{M}_{d}$ denotes the square-integrable irreducible representations of $M$ and for $\tau \in \hat{A}$, 
$\sigma \in \hat{M}_{d}, \sigma \times \tau$ is a representation of $M A N$ via $(\sigma \times \tau)($ man $)=\sigma(m) \tau(a)$. For $\sigma \in \hat{M}_{d}, \tau \in \hat{A}$ let $\pi(\sigma, \tau)=\operatorname{Ind}_{P_{1}}^{G} \sigma \times \tau$.

THEOREM 1.

$$
\pi\left(\sigma_{1}, \tau_{1}\right) \otimes \pi\left(\sigma_{2}, \tau_{2}\right) \simeq \operatorname{Ind}_{M A}^{G}\left(\sigma_{1} \otimes \sigma_{2}\right)\left(\tau_{1} \tau_{2}\right)
$$

where $\sigma_{1} \in \hat{M}_{d}, \tau_{i} \in \hat{A}, i=1,2$.

Proof. Since $P_{1}, P_{2}$ are associate parabolic subgroups, we have (see [11, p. 473]) $\operatorname{Ind}_{P_{1}}^{G} \sigma_{2} \times \tau_{2} \simeq \operatorname{Ind}_{P_{2}}^{G} \sigma_{2} \times \tau_{2}$ and we may apply Mackey's tensor product theorem with $G_{i}=P_{i}, i=1,2 . P_{1}, P_{2}$ are discretely related, there is exactly one double coset of positive measure in $G$, and if we take $e$ as a representative for this double coset, we have $P_{1} \cap P_{2}=M A$. Thus

$$
\begin{aligned}
\pi\left(\sigma_{1}, \tau_{1}\right) \otimes \pi\left(\sigma_{2}, \tau_{2}\right) & \simeq \operatorname{Ind}_{P_{1}}^{G} \sigma_{1} \times \tau_{1} \otimes \operatorname{Ind}_{P_{2}}^{G} \sigma_{2} \times \tau_{2} \\
& \simeq \operatorname{Ind}_{M A}^{G}\left(\sigma_{1} \otimes \sigma_{2}\right)\left(\tau_{1} \tau_{2}\right) .
\end{aligned}
$$

Thus the problem of decomposing the tensor product of two continuous series representations arising from the same cuspidal parabolic subgroup can be solved once one knows how to decompose $\sigma_{1} \otimes \sigma_{2}$ for $\sigma_{1}, \sigma_{2} \in \hat{M}_{d}$ and how to decompose $\operatorname{Ind}_{M A}^{G} L$ for all $L \in(M A)^{\wedge}$. However, there is no reason, a priori, to expect that either of these last two problems is any easier than the one we started with. In what follows, we show to what extent these problems can be dealt with when $P$ is a minimal parabolic subgroup and in particular when $G$ has real-rank one.

THEOREM 2. Let $P=M A N$ be a minimal parabolic subgroup. Then for $\sigma \in \hat{M}, \tau_{1}, \tau_{2} \in \hat{A}$ we have

$$
\operatorname{Ind}_{M A}^{G} \sigma \times \tau_{1} \simeq \operatorname{Ind}_{M A}^{G} \sigma \times \tau_{2} .
$$

Proof. Let $G$ have real-rank $l \geqslant 1$ and let $\alpha_{1}, \cdots, \alpha_{l}$ denote the simple (restricted) roots of $\boldsymbol{g}$. Since the simple roots form a basis for $a^{*}$ and $\hat{A} \approx a^{*}$, any $\tau^{\prime} \in \hat{A}$ is of the form $\tau^{\prime}(a)=e^{i \beta(H)}$ where $\beta=\Sigma_{i=1}^{l} m_{i} \alpha_{i}$, $m_{i} \in \mathbf{R}$, and $a=\exp H, H \in a$. Let $\tau^{j}(a)=e^{i m_{j} \alpha_{j}(H)}$ for $j=1, \cdots, l$ and $\tau^{0}=1$ where 1 is the 1 -dimensional identity representation of $A$ (i.e., the representation corresponding to $0 \in a^{*}$ ). Then $\boldsymbol{j}^{j} \in \hat{A}, i=0,1, \cdots, l$, and $\tau^{\prime}=\Sigma_{i=0}^{l} \tau^{j}$. We shall show that

$$
\begin{aligned}
\operatorname{Ind}_{M A}^{G} \sigma \times \tau^{\prime} \simeq \operatorname{Ind}_{M A}^{G} \sigma \times \prod_{i=0}^{L} \tau^{i} \simeq \cdots & \\
& \simeq \operatorname{Ind}_{M A}^{G} \sigma \times \tau^{1} \simeq \operatorname{Ind}_{M A}^{G} \sigma \times 1 .
\end{aligned}
$$


It clearly suffices to show

$$
\operatorname{Ind}_{M A}^{G} \sigma \times \tau \simeq \operatorname{Ind}_{M A}^{G} \sigma \times \prod_{i=0}^{i-1} \tau^{i}
$$

where $\tau=\Pi_{i=0}^{j} \tau^{i}$ and $j=1, \cdots, l$. Fix $\alpha=\alpha_{j}$ and let

$$
\mathfrak{n}_{\alpha}=\sum_{k>1} \mathfrak{g}_{k \alpha}, \quad \mathfrak{v}_{\alpha}=\theta \mathfrak{n}_{\alpha}=\sum_{k>1} g_{-k \alpha}, \quad \tau_{-}=\prod_{i=0}^{i} \tau^{i}
$$

Then, as in [10, p. 399], the subalgebra $8^{\alpha}$ of 8 generated by $\mathfrak{n}_{\alpha}$ and $\mathfrak{b}_{\alpha}$ is of the form $g^{\alpha}=a_{\alpha} \oplus \mathfrak{m}_{\alpha} \oplus \mathfrak{n}_{\alpha} \oplus \mathfrak{v}_{\alpha}$ where $\mathfrak{m}_{\alpha} \subseteq \mathfrak{m}=L A(M)$ and $a_{\alpha}=\mathbf{R} \cdot H_{\alpha}$. So $g^{\alpha}$ is a real-rank one semisimple subalgebra of $g$. Let $q=$ $8_{\alpha}$ or $q=8_{2 \alpha}$ according to whether $8_{2 \alpha}=\{0\}$ or not. If $Q=\exp q$, then $Q$ is an abelian subgroup of $N$ such that $M A$ leaves $Q$ invariant. In fact, from the lemma of $\S 4$, we know that $\exp \left(\mathrm{m}_{\alpha} \oplus \mathrm{a}_{\alpha}\right)$ will act on $Q-$ $\{e\}$ in two orbits or one orbit depending upon whether $\operatorname{dim} Q=1$ or not. If $\sigma \in \hat{M}=\hat{M}_{d}$ (M is compact), then (*) will follow by induction in stages [12, p. 113] once we show

$$
\pi_{\tau}=\operatorname{Ind}_{M A}^{M A} Q_{\sigma \times \tau} \simeq \operatorname{Ind}_{M A}^{M A} Q \sigma \times \tau_{-}=\pi_{-} .
$$

We first say something about the action of $M A$ on $q^{*}$ (via the coadjoint representation) and then define a function $D: q^{*} \rightarrow \Pi$ which is to satisfy the important identity

$$
D\left((m a)^{-1} \cdot \phi\right)=D(\phi) r^{j}(a) \text { for all } \phi \in q^{*}-\{0\}, m a \in M A .
$$

In the following, we let $k \alpha$ be $2 \alpha$ or $\alpha$ according to whether $q=$ $\mathbf{g}_{2 \alpha}$ or not. Let $(\cdot, \cdot)$ be an $\operatorname{Ad}(M)$-invariant inner product on $q$ and note that $(a \cdot X, Y)=(X, a \cdot Y)=e^{k \alpha(H)}(X, Y)$ where $a=\exp H, H \in a$, and $X, Y \in q$. Define a map $U: q^{*} \rightarrow q$ via $\phi \mapsto X_{\phi}$ where $\phi(X)=\left(X, X_{\phi}\right)$ for $X \in q$. Then $U$ is a vector space isomorphism (and so Borel). By definition we have $(m a \cdot \phi) X=\phi\left((m a)^{-1} \cdot X\right)$. So for all $X \in q$ we have

$$
\begin{aligned}
\left(X, X_{m a \cdot \phi}\right) & =(m a \cdot \phi)(X)=\phi\left(\left(m^{-1} a^{-1}\right) \cdot X\right) \\
& =\left(m^{-1} a^{-1} \cdot X, X_{\phi}\right)=\left(a^{-1} \cdot X, m \cdot X_{\phi}\right)=\left(X, m a^{-1} \cdot X_{\phi}\right),
\end{aligned}
$$

i.e., $X_{m a \circ \phi}=m a^{-1} \cdot X_{\phi}$. Note that if we define $\left(\phi_{1}, \phi_{2}\right)_{*}=\left(X_{\phi_{1}}, X_{\phi}\right)$ for $\phi_{1}, \phi_{2} \in q^{*}$, then $(\cdot, \cdot)^{*}$ is an $\operatorname{Ad}^{*}(M)$-invariant inner product on $q^{*}$. Let $\Phi_{0}$ be fixed in $q^{*}-\{0\}$.

If $\operatorname{dim} q>1$, then we know that $M A$ acts transitively on $q-\{0\}$.

Let $\phi \in q^{*}-\{0\}$ and choose $m a^{-1} \in M A \ni m a^{-1} \cdot X_{\phi_{0}}=X_{\phi}$. Then

$$
\begin{aligned}
\left(m a \cdot \phi_{0}\right)(X) & =\phi_{0}\left(m^{-1} a^{-1} \cdot X\right)=\left(m^{-1} a^{-1} \cdot X, X_{\phi_{0}}\right) \\
& =\left(X, m a^{-1} \cdot X_{\phi_{0}}\right)=\left(X, X_{\phi}\right)=\phi(X)
\end{aligned}
$$


and so $m a \cdot \phi_{0}=\phi$. Thus $M A$ also acts transitively on $q^{*}-\{0\}$. So for $\operatorname{dim} q>1$, define $D: q^{*} \rightarrow \Pi$ by $D(0)=1, D\left((m a)^{-1} \cdot \phi_{0}\right)=\tau^{j}(a)$. Note that $D$ is defined on all of $q^{*}$ and is a Borel function. Note also that

(1) $D$ is well-defined.

Suppose $(m a)^{-1} \cdot \phi_{0}=\phi_{0}$ with $a=\exp H, H \in a$. Since $\left(a \cdot \phi_{0}\right)(X)$ $=\left(a^{-1} \cdot X, X_{\phi_{0}}\right)=e^{-k \alpha(H)}\left(X, X_{\phi_{0}}\right)=e^{-k \alpha(H)} \phi_{0}(X)$, we have $a \cdot \phi_{0}=$ $e^{-k \alpha(H)} \phi_{0}$. Let $\|\phi\|=(\phi, \phi)_{*}^{1 / 2}$. Then $\left\|m^{-1} a^{-1} \cdot \phi_{0}\right\|=\left\|a^{-1} \cdot \phi_{0}\right\|=$ $e^{k \alpha(H)}\left\|\phi_{0}\right\|=\left\|\phi_{0}\right\|$ implies that $k \alpha(H)=0$. Hence $\tau^{j}(a)=e^{i m_{j} k \alpha(H)}=1$ and $D$ is well-defined.

(2) $D\left((m a)^{-1} \cdot \phi\right)=D(\phi) \tau^{j}(a)$ for $\phi \in \mathbb{g}_{k \alpha}^{*}-\{0\}, m a \in M A$. Let $\phi \in$ $q^{*}-\{0\}$ and choose $m_{1} a_{1} \in M A\left(m_{1} a_{1}\right)^{-1} \cdot \phi_{0}=\phi$. Then

$$
\begin{aligned}
D\left((m a)^{-1} \cdot \phi\right) & =D\left(\left(m m_{1} a a_{1}\right)^{-1} \cdot \phi_{0}\right)=\tau^{j}\left(a a_{1}\right) \\
& =\tau^{j}(a) \tau^{j}\left(a_{1}\right)=D(\phi) \tau^{j}(a) .
\end{aligned}
$$

Now suppose $\operatorname{dim} q=1$. We know $\exp \left(a_{\alpha} \oplus \mathfrak{m}_{\alpha}\right)$ acts on $q-\{0\}$ in two orbits (although it is conceivable that $M A$ acts in one orbit), in fact, we know that $a \cdot X=e^{k \alpha(H)} X$ for $X \in q-\{0\}, a=\exp H, H \in \mathfrak{a}$ and that $a \cdot \phi=e^{-k \alpha(H)} \phi$ for $\phi \in q^{*}-\{0\}$. So $A$ acts on $q^{*}-\{0\}$ in two orbits. Define $D: q^{*} \rightarrow \Pi$ by $D(0)=1, D\left((m a)^{-1} \cdot\left( \pm \phi_{0}\right)\right)=\tau^{j}(a)$. Then $D$ is Borel, defined on all of $q^{*}$, and

(1) $D$ is well-defined:

Suppose $(m a)^{-1} \cdot\left( \pm \phi_{0}\right)= \pm \phi_{0}$. Then, as above, $k \alpha(H)=0$ and $\tau^{j}(a)$ $=1$.

(2) $D\left((m a)^{-1} \cdot \phi\right)=D(\phi) \tau^{j}(a)$ for $\phi \in q^{*}-\{0\}, m a \in M A$. Let $\phi \in$ $q^{*}-\{0\}$ and choose $a_{1} \in A \ni a_{1}^{-1} \cdot\left( \pm \phi_{0}\right)=\phi$. Then

$$
D\left((m a)^{-1} \cdot \phi\right)=D\left(\left(m a a_{1}\right)^{-1} \cdot\left( \pm \phi_{0}\right)\right)=\tau^{j}\left(a a_{1}\right)=D(\phi) \tau^{j}(a) .
$$

Let $d X$ denote Lebesgue measure on $q$ and choose Haar measure $d q$ on $Q$ such that $\int_{Q} f(q) d q=\int_{q} f(\exp X) d X$ for $f \in L^{1}(Q, d q)$. If we let $\psi: L^{2}(Q, d q) \rightarrow L^{2}(q, d X)$ via $(\psi f)(X)=f(\exp X)$, then $\psi$ is a unitary operator between $L^{2}(Q)$ and $L^{2}(q)$. For $m a \in M A$, let $\Delta_{k \alpha}(m a)$ denote the modulus of the automorphism $q \mapsto(m a) q(m a)^{-1}, q \in Q$. As in [10, p. 392], since $m \mapsto \Delta_{k \alpha}(m)$ defines a continuous homomorphism of the compact group $M / \operatorname{center}(G)$ into the multiplicative group of positive reals, we have that $\Delta_{k \alpha}(m)=1$, for all $m \in M$. Now let $a=\exp H, H \in \mathfrak{a}$ and $\operatorname{dim} q=c_{k \alpha}$. Then

$$
\begin{aligned}
\Delta_{k \alpha}(a) & =\operatorname{det}(\operatorname{Ad}(a))=\operatorname{det}(\exp \operatorname{ad} H)=\exp (\operatorname{tr} \operatorname{ad} H) \\
& =\exp \left(c_{k \alpha} k \alpha(H)\right) .
\end{aligned}
$$


So

$$
\int_{Q} f(q) d q=\Delta_{k \alpha}(a) \int_{Q} f\left((m a) q(m a)^{-1}\right) d q
$$

Now

$$
\begin{aligned}
\int_{q}(\Psi f)(\operatorname{Ad}(m a) X) d X & =\int_{q} f(\exp \operatorname{Ad}(m a) X) d X \\
& =\int_{Q} f\left((m a) q(m a)^{-1}\right) d q \\
& =\Delta_{k \alpha}\left(a^{-1}\right) \int_{Q} f(q) d q=\Delta_{k \alpha}\left(a^{-1}\right) \int_{q}(\Psi f)(X) d X .
\end{aligned}
$$

Thus the modulus of $X \mapsto \operatorname{Ad}(m a) X$ is also $\Delta_{k \alpha}(a)=e^{c_{k \alpha} k \alpha(H)}$.

We now prove (**). If $f \in L^{2}\left(Q, H_{\sigma}\right)$, then

$$
\pi_{\tau}(\operatorname{man}) f(q)=\sigma(m) \tau(a) f\left(m^{-1} a^{-1} q a m n\right) \Delta_{k \alpha}^{-1 / 2}(a)
$$

and

$$
\pi_{-}(m a n) f(q)=\sigma(m) \prod_{i=0}^{i-1} \tau^{i}(a) f\left(m^{-1} a^{-1} g a m n\right) \Delta_{k \alpha}^{-1 / 2}(a)
$$

where man $\in M A Q$. Now let $\Psi: L^{2}\left(Q, H_{\sigma}\right) \rightarrow L^{2}\left(q, H_{\sigma}\right)$ by $(\Psi f)(X)=$ $f(\exp X)$. Then $\Psi$ is a unitary operator and for $h \in L^{2}\left(q, H_{\sigma}\right)$ with $\Psi f=$ $h$ we have

$$
\begin{aligned}
\tilde{\pi}_{\tau}(\text { man }) h(X) & \equiv\left(\Psi \pi_{\tau}(\text { man }) \psi^{-1} h\right)(X)=\left(\psi \pi_{\tau}(\text { man }) f\right)(X) \\
& =\pi_{\tau}(\text { man }) f(\exp X) \\
& =\sigma(m) \tau(a) f\left(m^{-1} a^{-1}(\exp X) a m \exp X_{n}\right) \Delta_{k \alpha}^{-1 / 2}(a), \quad n=\exp X_{n}, \\
& =\sigma(m) \tau(a) f\left(\left(\exp \operatorname{Ad}(m a)^{-1} X\right) \exp X_{n}\right) \Delta_{k \alpha}^{-1 / 2} \\
& =\sigma(m) \tau(a) f\left(\exp \left(\operatorname{Ad}(m a)^{-1} X+X_{n}\right)\right) \Delta_{k \alpha}^{-1 / 2}(a) \\
& =\sigma(m) \tau(a) h\left(\operatorname{Ad}(m a)^{-1} X+X_{n}\right) \Delta_{k \alpha}^{-1 / 2}(a) .
\end{aligned}
$$

Similarly

$$
\tilde{\pi}_{-}(\operatorname{man}) h(x)=\sigma(m) \prod_{i=0}^{j-1} \tau^{i}(a) h\left(\operatorname{Ad}(\operatorname{ma})^{-1} X+X_{n}\right) \Delta_{k \alpha}^{-1 / 2}(a) .
$$

For $X \in q, f \in L^{2}\left(q, H_{\sigma}\right)$, and $\phi \in q^{*}$ we have

$$
(F f)(\phi)=\int_{q} f(X) e^{i \phi(X)} d X,
$$

the operator-valued Fourier transform on $q$. We now use $F$ to realize $\tilde{\pi}_{\tau}$ and $\tilde{\pi}_{-}$on $L^{2}\left(q^{*}, H_{\sigma}\right)$. Let $H \in L^{2}\left(q^{*}, H_{\sigma}\right)$ with $F h=H$. 


$$
\begin{aligned}
\hat{\pi}_{\tau}(\text { man }) H(\phi) & =\left(F \tilde{\pi}_{\tau}(\text { man }) F^{-1} H\right)(\phi) \\
& =\int_{q} \tilde{\pi}_{\tau}(\text { man }) h(X) e^{i \phi(X)} d X \\
& =\int_{q} \sigma(m) \tau(a) h\left(\operatorname{Ad}(m a)^{-1} X+X_{n}\right) \Delta_{k \alpha}^{-1 / 2}(a) e^{i \phi(X)} d X \\
& =\sigma(m) \tau(a) \int_{q} h\left(X+X_{n}\right) \exp \left\{i \int(\operatorname{Ad}(m a) X)\right\} \Delta_{k \alpha}^{1 / 2}(a) d X \\
& =\sigma(m) \tau(a) \int_{q} h(X) \exp \left\{i \phi\left(\operatorname{Ad}(m a)\left(X-X_{n}\right)\right)\right\} \Delta_{k \alpha}^{1 / 2}(a) d X \\
& =\sigma(m) \tau(a) \Delta_{k \alpha}^{1 / 2}(a) \exp \left\{-i\left((m a)^{-1} \cdot \phi\right)\left(X_{n}\right)\right\} H\left((m a)^{-1} \cdot \phi\right) .
\end{aligned}
$$

Similarly,

$$
\hat{\pi}_{-}(\operatorname{man}) H(\phi)=\sigma(m) \prod_{i=0}^{j-1} \tau^{i}(a) \Delta_{k \alpha}^{1 / 2}(a) \exp \left\{-i\left((m a)^{-1} \cdot \phi\right)\left(X_{n}\right)\right\} H\left((m a)^{-1} \cdot \phi\right) .
$$

Now define $B: L^{2}\left(q^{*}, H_{\sigma}\right) \rightarrow L^{2}\left(q^{*}, H_{\sigma}\right)$ by $g(\phi) \rightarrow D(\phi) g(\phi)$. Then $B$ is clearly unitary and for $B^{-1} g=G$ we have

$$
\begin{aligned}
& \left(B \hat{\pi}_{\tau}(\text { man }) B^{-1} g\right)(\phi)=\left(B \hat{\pi}_{\tau}(\text { man }) G\right)(\phi)=D(\phi) \hat{\pi}_{\tau}(\text { man }) G(\phi) \\
& =D(\phi) \sigma(m) \tau(a) \Delta_{k \alpha}^{1 / 2}(a) \exp \left\{-i\left((m a)^{-1} \cdot \phi\right)\left(X_{n}\right)\right\} G\left((m a)^{-1} \cdot \phi\right) \\
& =D(\phi) \sigma(m) \tau^{i}(a) \prod_{i=0}^{j-1} \tau^{i}(a) \Delta_{k \alpha}^{1 / 2}(a) \exp \left\{-i\left((m a)^{-1} \cdot \phi\right)\left(X_{n}\right)\right\} G\left((m a)^{-1} \cdot \phi\right) \\
& =D\left((m a)^{-1} \cdot \phi\right) \prod_{i=0}^{j-1} \tau^{i}(a) \sigma(m) \Delta_{k \alpha}^{1 / 2}(a) \exp \left\{-i\left((m a)^{-1} \cdot \phi\right)\left(X_{n}\right)\right\} G\left((m a)^{-1} \cdot \phi\right) \\
& \text { for } \phi \neq 0 \text {. } \\
& =\sigma(m) \prod_{i=0}^{j-1} \tau^{i}(a) \Delta_{k \alpha}^{1 / 2}(a) e^{-i\left((m a)^{-1} \diamond \phi\right)\left(X_{n}\right)} g\left((m a)^{-1} \cdot \phi\right), \quad \phi \neq 0 \\
& =\hat{\pi}_{-}(\operatorname{man}) g(\phi), \quad \phi \neq 0 .
\end{aligned}
$$

So $\pi_{\tau} \simeq \pi_{-}$and Theorem 2 has been proven.

Now let $G$ be a connected semisimple Lie group with finite center and Iwasawa decomposition $K A N$. If $M$ is the centralizer of $A$ in $K$, then $M$ is compact and $P=M A N$ is a minimal parabolic subgroup of $G$. Thus $\sigma_{1} \otimes$ $\sigma_{2} \simeq \Sigma_{j} b_{j} \tau_{j}$ where $\sigma_{j} \in \hat{M}=\hat{M}_{d}$, the sum is finite, and the $b_{j}$ 's are called the Clebsch-Gordan coefficients for $\sigma_{1}, \sigma_{2}$. Theorem 1 now states that

$$
\pi\left(\sigma_{1}, \tau_{1}\right) \otimes \pi\left(\sigma_{2}, \tau_{2}\right) \simeq \sum_{j}^{\infty} b_{j} \operatorname{Ind}_{M A}^{G}\left(\sigma_{j} \times \tau\right), \quad \tau=\tau_{1} \tau_{2},
$$


and so the problem of decomposing the tensor product of two principal series representations reduces to that of knowing $\operatorname{Ind}_{M A}^{G} L$ for all $L \in(M A)^{\wedge}$. Theorem 2 states that inducing from $M A$ is independent of the character on $A$ and so if we could determine $\operatorname{Ind}_{M A}^{G} L$ for $\mu_{C}$-almost all $L \in(M A)^{\wedge}$, then we would know $\operatorname{Ind}_{M A}^{G} L$ for all $L \in(M A)^{\wedge}$. By Anh's reciprocity theorem, the problem of finding $\operatorname{Ind}_{M A}^{G} L$ for $\mu_{C}$-almost all $L \in(M A)^{\wedge}$ is equivalent to finding $(\pi)_{M A}$ (note that the measure in this decomposition must be absolutely continuous with respect to $\mu_{C}$ ) for $\mu_{G}$-almost all $\pi \in \hat{G}$. We now show to what extent we can solve this last problem when $G$ has real-rank one.

From now on, we assume that $G$ is a connected semisimple real-rank one Lie group with finite center. From Theorems 1 and 2, we know how to decompose the tensor product of two principal series representations of $G$ once we know how to decompose $(\pi)_{M A}$ for $\mu_{G}$-almost all $\pi \in \hat{G}$, i.e., for almost all principal series and all discrete series representations of $G$.

We now proceed to find $(\pi)_{M A}$ when $\pi$ is a principal series representation by using Mackey's subgroup theorem.

Lemma 3. Let MA act on $V$ by inner automorphism. Then up to a set of measure zero, this action corresponds to the canonical action (on the right) of $M A$ on $P \backslash$. If $S \subseteq V$ is a cross-section for $V / M A$, then up to a set of measure zero $S$ also serves as a cross-section for $P \backslash G / M A$.

Proof. Let $g=b v, b \in P, v \in V$ and $m a \in M A$. Then $m a \cdot M A N g=M A N b u m a=N M A v m a=N M A(m a)^{-1} v(m a)=M A N(m a)^{-1} v(m a)$ and so the first part is clear. Now define a map $\Psi: V / M A \rightarrow P \backslash P V / M A$ via $\Psi: \quad O \rightarrow D$ where $O \in V / M A, D$ is the double coset containing $v$, and $v$ is any point in $O$. That $\Psi$ is well defined and onto is clear. Now suppose $v_{1}, v_{2} \in V$ lie in the same double coset. Then $v_{2}=n m^{\prime} a^{\prime} v_{1} m a$ for some $m, m^{\prime} \in M, a, a^{\prime} \in A$, and $n \in N$. So $v_{2}=n\left(m^{\prime} a^{\prime} m a\right)\left((m a)^{-1} v_{1} m a\right)$ and since elements in $N M A V$ are expressed uniquely, we have $(m a)^{-1} v_{1} m a=v_{2}$ and $v_{1}, v_{2}$ lie in the same orbit in $V / M A$. Thus $\Psi$ is also 1-1 (in fact a Borel isomorphism) and the second part is also clear.

We now fix $X_{-\alpha} \in 8_{-\alpha}-\{0\}$ and $X_{-2 \alpha} \in 8_{-2 \alpha}-\{0\}$.

Lemma 4. Let $V^{\prime}=\left\{\exp W \exp T=\exp (W+T): W \in \mathfrak{g}_{-\alpha}^{x}, T \in \boldsymbol{g}_{-2 \alpha}^{x}\right\}$, and set

$$
S= \begin{cases}\left\{\exp \left( \pm X_{-\alpha}\right)\right\} & \text { if } \operatorname{dim} 8_{-2 \alpha}=0, \operatorname{dim} 8_{-\alpha}=1, \\ \left\{\exp \left(X_{-\alpha}\right)\right\} & \text { if } \operatorname{dim} 8_{-2 \alpha}=0, \operatorname{dim} 8_{-\alpha}>1, \\ \left\{\exp \left(t X_{-\alpha} \pm Y_{-2 \alpha}\right): t>0\right\} & \text { if } \operatorname{dim} 8_{-2 \alpha}=1, \\ \left\{\exp \left(t X_{-\alpha}+Y_{-2 \alpha}\right): t>0\right\} & \text { if } \operatorname{dim} 8_{-2 \alpha}>1 .\end{cases}
$$


Then $S$ is a Borel cross-section in $V^{\prime}$ for the orbits under the action of $M A$.

Proof. As discussed in $\$ 4$, we may assume that $G$ is simple. For the classical simple real-rank one Lie groups it is immediate, by looking at the realizations of these groups and the action of $M A$ on $V$ given in $\S 4$, that the above sets form cross-sections for $V / M A$. That essentially the same arguments hold for the exceptional case, $F_{4,9}$, is not so clear and we thank $K$. Johnson for forwarding a paper [6] which made clear some of the underlying results in Kostant [9]. So now let $G=\mathrm{F}_{4,9}$ and suppose $\exp \left(t_{1} X_{-\alpha}+Y_{-2 \alpha}\right), \exp \left(t_{2} X_{-\alpha}+Y_{-2 \alpha}\right)$ lie in the same orbit in $V / M A$. Then there exists $c=m a \in M A$ such that $c^{-1} \exp \left(t_{1} X_{-\alpha}+Y_{-2 \alpha}\right) c=\exp \left(t_{2} X_{-\alpha}+Y_{-2 \alpha}\right)$. So $a=e, m \in M_{Y_{-2 \alpha}}=$ $\left\{m \in M: m \cdot Y_{-2 \alpha}=Y_{-2 \alpha}\right\}$, and $\operatorname{Ad}(m) t_{1} X_{-\alpha}=t_{2} X_{-\alpha}$, i.e., $\operatorname{Ad}(m) X_{-\alpha}=$ $\left(t_{2} / t_{1}\right) X_{-\alpha}$ and $t_{1}=t_{2}$. So $S$ meets any orbit in $V / M A$ in at most one point. What we must show is that $S$ does indeed meet each orbit.

We know that $K=\operatorname{Spin}(9), M=\operatorname{Spin}(7), \operatorname{dim} 8_{-\alpha}=8, \operatorname{dim} g_{-2 \alpha}=7^{\circ}$, and that $M$ acts on both $8_{-\alpha}$ and $8_{-2 \alpha}$. From the lemma of $\S_{4}$, we see that $M$ acts transitively on spheres in both $g_{-\alpha}, g_{-2 \alpha}$ and hence that $M$ must act irreducibly on $8_{-\alpha}$ and $8_{-2 \alpha}$. If we put an Ad(M)-invariant inner product on $b=g_{-\alpha} \oplus g_{-2 \alpha}$, then we have that $M$ acts as SO(7) on $g_{-2 \alpha}$ and as the Spin respresentation on $8_{-\alpha}$ (since these are the only irreducible representations of $M$ in these dimensions). If we let $S_{-\alpha}, S_{-2 \alpha}$ denote the unit spheres in $8_{-\alpha}, g_{-2 \alpha}$ respectively, then we obtain an action of $M$ on $S_{-\alpha} \oplus$ $S_{-2 \alpha}$ for which we now show: given $\left(u_{1}, v_{1}\right),\left(u_{2}, v_{2}\right) \in S_{-\alpha} \oplus S_{-2 \alpha}$, there is an $m \in M$ such that $m \cdot\left(u_{1}, v_{1}\right)=\left(u_{2}, v_{2}\right)$. Since $M=\operatorname{Spin}(7)$ is transitive on $S_{-2 \alpha}$, we may assume $v_{1}=v_{2}=v$. The subgroup of $\operatorname{Spin}(7)$ which leaves $v$ fixed is $\operatorname{Spin}(6) \approx \mathrm{SU}(4)$ and when the spin representation of Spin (7) acting on $g_{-\alpha}$ is restricted to Spin (6), we obtain the irreducible representation of $\mathrm{SU}(4)$ on $\mathbf{C}^{4} \approx \mathbf{R}^{8}$. Since $\mathrm{SU}(4)$ is transitive on $S^{7}$, we obtain our result.

Now we know that $M A$ acts transitively on $g_{-2 \alpha}$ and $M_{Y_{-2}} A$ acts transitively on $g_{-\alpha}$. So let $(W, T) \in \mathfrak{b}$, with $0 \neq W \in g_{-\alpha}, 0 \neq T \in g_{-2 \alpha}$. Choose $c \in M A$ such that $\operatorname{Ad}(c) T=Y_{-2 \alpha}$ and then choose $m \in M_{Y_{-2 \alpha}}$ such that $\operatorname{Ad}(m)(\operatorname{Ad}(c) W)=t X_{-\alpha}, t>0$. Then $m c \in M A$ and $\operatorname{Ad}(m c)(W, T)$ $=\left(t X_{-\alpha}, Y_{-2 \alpha}\right)$ as desired.

Note. (1) Since $\operatorname{Ad}(m) t X=t \operatorname{Ad}(m) X, t \in \mathbf{R}, m \in M, X \in \mathfrak{v}$, the stability subgroup at any point in $S \subseteq V$ under the action of $M A$ is equal to

$$
\left\{m \in M: \quad \operatorname{Ad}(m) Y_{-2 \alpha}=Y_{-2 \alpha}, \quad \operatorname{Ad}(m) X_{-\alpha}=X_{-\alpha}\right\}=M_{0}
$$

and so along our cross-section $S$ all the stability subgroups are equal to $M_{0}$.

(2) Aside from a set of Haar measure zero in $G, P \backslash G / M A$ can be identified with $S$ and so the following is clear: 
LEMMA 5. $M A$ and $M A N$ are regularly related.

Lemma 6. For $s \in S, M A \cap s^{-1} M A N s=M_{0}$.

ProOF. In light of the correspondence between the action of $M A$ on $V$ by inner automorphism and the natural action of $M A$ on $P \backslash P V$, it suffices to prove the following:

Claim. Suppose $H_{2}$ acts on $H_{1} \backslash G$ on the right. Then $H_{1} \backslash G$ is the union of orbits $O_{i}$. Let $x_{i} \in O_{i}$ and $M_{x_{i}}=$ the isotropy subgroup at $x_{i}$. Then $M_{x_{i}}=H_{2} \cap x_{i}^{-1} H_{1} x_{i}$.

Proof of claim.

$$
\begin{aligned}
M_{x_{i}} & =\left\{h \in H_{2}: h \cdot\left(H_{1} x_{i}\right)=H_{1} x_{i}\right\}=\left\{h \in H_{2}: H_{1} x_{i} h x_{i}^{-1}=H_{1}\right\} \\
& =\left\{h \in H_{2}: h \in x_{i}^{-1} H_{1} x_{i}\right\}=H_{2} \cap x_{i}^{-1} H_{1} x_{i} .
\end{aligned}
$$

LEMMA 7. Let $\eta \in M_{v}=\left\{m \in M: m^{-1} v m=v\right\}$ for $v \in V$ and let $L \in M A$. Then $\eta \rightarrow L_{\left(v \eta v^{-1}\right)}$ is $(L)_{M_{v}}$.

Proof. $\eta \in M_{v} \Rightarrow u \eta v^{-1}=\eta$ and $L_{\left(v \eta v^{-1}\right)}=L_{(\eta)}$.

Now define

$$
\#(S)= \begin{cases}\text { cardinality of } S, & \text { if the cardinality of } S \text { is finite, } \\ \infty, & \text { otherwise. }\end{cases}
$$

THEOREM 8. Let $S, M_{0}$ be as above, $\sigma \in \hat{M}, \tau \in \hat{A}$, and ${ }^{A} R$ denote the regular representation of $A$. Then

$$
(\pi(\sigma, \tau))_{M_{A}} \simeq \#(S) \operatorname{Ind}_{M_{0}}^{M A}(\sigma)_{M_{0}} \simeq \#(S)\left(\operatorname{Ind}_{M_{0}}^{M}(\sigma)_{M_{0}} \times{ }^{A} R\right) .
$$

Proof. We apply Mackey's subgroup theorem by identifying the collection of $P: M A$ double cosets in $G$ with $S$ and choosing any admissible measure $\nu$ on $S$ (note that when $\#(S)=\infty$, there are no orbits of positive measure in $V$ and so $\nu$ will be nonatomic). Then

$$
\begin{aligned}
(\pi(\sigma, \tau))_{M A} & \simeq \int_{S}^{\oplus} \operatorname{Ind}_{M_{0}}^{M A}(\sigma)_{M_{0}} d v(s) \simeq \#(S) \operatorname{Ind}_{M_{0}}^{M A}(\sigma)_{M_{0}} \\
& \simeq \#(S) \operatorname{Ind}_{M_{0} \times\{e\}}^{M \times A}(\sigma)_{M_{0}} \times 1 \simeq \#(S)\left(\operatorname{Ind}_{M_{0}}^{M}(\sigma)_{M_{0}} \times \operatorname{Ind}_{\{e\}}^{A} 1\right) \\
& \simeq \#(S)\left(\operatorname{Ind}_{M_{0}}^{M}(\sigma)_{M_{0}} \times{ }^{A} R\right) .
\end{aligned}
$$

Note that in determining $\operatorname{Ind}_{M_{0}}^{M}(\sigma)_{M_{0}}$, we may use the compact version of the reciprocity theorem and that the multiplicity of a $\sigma^{\prime} \times \tau^{\prime} \in M A$ occurring in $(\pi(\sigma, \tau))_{M A}$ does not depend on $\tau^{\prime}$. So the above decomposition can be written

$$
(\pi(\sigma, \tau))_{M A} \simeq \#(S) \int_{\hat{C}}^{\oplus} n\left(\pi, \sigma^{\prime}\right)\left(\sigma^{\prime} \times \tau^{\prime}\right) d \mu_{C}\left(\sigma^{\prime}, \tau^{\prime}\right)
$$


where $\mu_{C}$ denotes Plancherel measure on $M A=\hat{C}$ and $n\left(\dot{\pi}, \sigma^{\prime}\right)$ can be explicitly computed (see Theorem 16).

Now let $\hat{G}_{d}$ denote the discrete series representations for $G$. We now attempt to find $(\pi)_{M A}$ for $\pi \in \hat{G}_{d}$. We first find $\left(\operatorname{Ind}_{K}^{G} \sigma\right)_{M A}$ for $\sigma \in \hat{K}$.

LEMma 9. The action of $M$ on $N$ by inner automorphism corresponds to the action of $M A$ on $K \backslash G$ on the right and if $S_{1} \subseteq N$ denotes a crosssection for $N / M$, then $S_{1}$ also serves as a cross-section for $K \backslash G / M A$.

Proof. Let $g=k n a, k \in K, a \in A, n \in N$ and $m a^{\prime} \in M A$. Then $m a^{\prime} \cdot K g=K n a m a^{\prime}=K m^{-1} n m a a^{\prime}$ and so the first part is clear. Define $\Psi$ : $N / M \rightarrow K \backslash G / M A$ by sending an orbit 0 to the double coset $D$, where $D$ is the double coset containing $v$, and $v$ is any point in 0 . Two points in the same orbit in $N / M$ clearly lie in the same $K: M A$ double coset and so $\Psi$ is well defined. That $\Psi$ is onto is clear. Now suppose $n_{1}, n_{2}$ lie in the same $K: M A$ double coset. Then $n_{2}=k n_{1} m a$ for some $k \in K, m a \in M A$. Thus $n_{2}=(\mathrm{km})\left(\mathrm{m}^{-1} n_{1} \mathrm{~m}\right) \mathrm{a}$ and since elements in $K N A$ are expressed uniquely, we have $m^{-1} n_{1} m=n_{2}$, i.e., $n_{1}, n_{2}$ are in the same orbit in $N / M$ and $\Psi$ is 1-1. Lemma 9 is now clear. and set

Lemma 10. Let $X_{\alpha}=\theta X_{-\alpha} \in g_{\alpha}-\{0\}, Y_{2 \alpha}=\theta Y_{-2 \alpha} \in g_{2 \alpha}-\{0\}$,

$$
S_{1}= \begin{cases}\left\{\exp \left(t X_{\alpha}\right): t \in \mathbf{R}\right\} & \text { if } \operatorname{dim} 8_{2 \alpha}=0, \operatorname{dim} 8_{\alpha}=1, \\ \left\{\exp \left(t X_{\alpha}\right): t \geqslant 0\right\} & \text { if } \operatorname{dim} 8_{2 \alpha}=0, \operatorname{dim} 8_{\alpha}>1, \\ \left\{\exp \left(t X_{\alpha}+s Y_{\alpha}\right): t \geqslant 0, s \in \mathbf{R}\right\} & \text { if } \operatorname{dim} 8_{2 \alpha}=1, \\ \left\{\exp \left(t X_{\alpha}+s Y_{\alpha}\right): t \geqslant 0, s \geqslant 0\right\} & \text { if } \operatorname{dim} 8_{2 \alpha}>1 .\end{cases}
$$

Then $S_{1}$ is a Borel cross-section in $N$ for the orbits under the action of $M$.

Proof. We proceed as in Lemma 4. Since the classical cases are again clear by inspection, we shall deal only with the exceptional case. First suppose $\exp \left(t_{1} X_{\alpha}+s_{1} Y_{\alpha}\right), \exp \left(t_{2} X_{\alpha}+s_{2} Y_{\alpha}\right)$ lie in the same orbit in $N / M$. Then there exists $m \in M$ such that $\operatorname{Ad}(m) t_{1} X_{\alpha}=\operatorname{Ad}(m) t_{2} X_{\alpha}, \operatorname{Ad}(m) s_{1} Y_{\alpha}=$ $\operatorname{Ad}(m) s_{2} Y_{\alpha}$ where $t_{1}, t_{2}, s_{1}, s_{2}$ are all $\geqslant 0$. Thus $t_{1}=t_{2}, s_{1}=s_{2}$, and $S_{1}$ meets any orbit in at most one point.

As in Lemma 4, $M$ acts transitively on spheres in $g_{2 \alpha}$ while $M_{Y_{\alpha}}$ acts transitively on spheres in $g_{\alpha}$. So for $(W, T) \in \mathfrak{n}=L A(N)$ we may choose $m \in M$ such that $\operatorname{Ad}(m) T=s Y_{\alpha}$ for some $s \geqslant 0$ and then an $m^{\prime} \in M_{Y_{\alpha}}$ such that $\operatorname{Ad}\left(m^{\prime}\right) \operatorname{Ad}(m) W=t X_{\alpha}$ for some $t \geqslant 0$. Then $m^{\prime} m \in M$ and $\operatorname{Ad}\left(m^{\prime} m\right)(W, T)=\left(t X_{\alpha}, s Y_{\alpha}\right)$ and hence $S_{1}$ meets every orbit in $N / M$.

Note. (1) The isotropy subgroup along $S_{1}$ are again all equal, and in each case we have $\#\left(S_{1}\right)=\infty$. 
(2) Since $\operatorname{Ad}(m) \theta X_{\alpha}=\theta\left(\operatorname{Ad} m X_{\alpha}\right)$ for $m \in M$ and $X_{\alpha} \in g_{k \alpha}, k=$ 1, 2, we have that $\left\{m \in M: \operatorname{Ad}(m) X_{\alpha}=X_{\alpha}\right\}=\left\{m \in M: \operatorname{Ad}(m) \theta X_{\alpha}=\theta X_{\alpha}\right\}$. So $M_{0}=\left\{m \in M\right.$ : $\left.\operatorname{Ad}(m) X_{-\alpha}=X_{-\alpha}, \operatorname{Ad}(m) Y_{-2 \alpha}=Y_{-2 \alpha}\right\}=\{m \in M$ : $\left.\operatorname{Ad}(m) X_{\alpha}=X_{\alpha}, \operatorname{Ad}(m) Y_{\alpha}=Y_{\alpha}\right\}=$ the isotropy subgroup (s) along $S_{1}$.

(3) We may identify $S_{1}$ with $K \backslash G / M A$ and so the following is clear.

LEMMA 11. $K$ and $M A$ are regularly related in $G$.

LEMMA 12. For $s \in S_{1}, M A \cap s^{-1} K s=M_{0}$.

Proof. Same as claim in Lemma 6.

THEOREM 13. Let $S_{1}, M_{0}$ be as above and $\sigma \in \hat{K}$. Then

$$
\left(\operatorname{Ind}_{K}^{G} \sigma\right)_{M_{A}} \simeq{ }^{\infty}\left(\operatorname{Ind}_{M_{0}}^{M}(\sigma)_{M_{0}} \times{ }^{A} R\right) .
$$

Proof. Mackey's subgroup theorem implies that

$$
\left(\operatorname{Ind}_{K}^{G} \sigma\right)_{M A} \simeq \int_{S_{1}}^{\oplus} \operatorname{Ind}_{M_{0}}^{M A}(\sigma)_{M_{0}} d v(s)
$$

where $\nu$ is any admissible measure on $S_{1}$. So as before

$$
\left(\operatorname{Ind}_{K}^{G} \sigma\right)_{M A} \simeq \#\left(S_{1}\right)\left(\operatorname{Ind}_{M_{0}}^{M}(\sigma)_{M_{0}} \times{ }^{A} R\right) \simeq \propto\left(\operatorname{Ind}_{M_{0}}^{M}(\sigma)_{M_{0}} \times{ }^{A} R\right) .
$$

LEMMA 14. $\pi_{0} \in \hat{G}_{d} \Rightarrow \exists \delta \in \hat{K} \ni \pi_{0}$ is contained in $\operatorname{Ind}_{K}^{G} \delta$.

Proof. We use Ahn's reciprocity theorem. We know that, for all $\pi \in \hat{G}$, $(\pi)_{K} \simeq \Sigma_{\delta \in \hat{K}} n(\pi, \delta) \delta$ and so, for all $\delta \in \hat{K}$, we have $\operatorname{Ind}_{K}^{G} \delta \simeq$ $\int_{\hat{G}}^{\oplus} n(\pi, \delta) \pi d \mu_{G}(\pi)$. Now there exists a $\delta \in \hat{K}$ for which $n\left(\pi_{0}, \delta\right) \neq 0$ (lest $\left.\left(\pi_{0}\right)_{K}=0\right)$ and since $\mu_{G}\left(\left\{\pi_{0}\right\}\right)>0$, we have found a $\delta \in \hat{K}$ for which $\pi_{0}$ is contained in $\operatorname{Ind}_{K}^{G} \delta$.

THEOREM 15. $\pi \in \hat{G}_{d} \Rightarrow(\pi)_{M A} \simeq \int_{\hat{C}}^{\oplus} n(\sigma, \tau, \pi)(\sigma \times \tau) d \mu_{C}(\sigma, \tau)$ where $\mu_{C}$ is Plancherel measure on $\hat{C}=M A-(M A)^{-}$and $n(\sigma, \tau, \pi) \in\{0,1,2, \cdots, \infty\}$.

Proof. By Lemma $14, \exists \delta \in \hat{K}$ such that $\pi$ is contained in $\operatorname{Ind}_{K}^{G} \delta$ and by Theorem 13

$$
\left(\operatorname{Ind}_{K}^{G} \delta\right)_{M A} \simeq \int_{\hat{C}}^{\oplus} n^{\prime}(\sigma, \delta, \pi)(\sigma \times \tau) d \mu_{C}(\sigma, \tau)
$$

where $n^{\prime}(\sigma, \delta, \pi) \in\{0, \infty\}$. So by $[4$, p. 273] we have

$$
(\pi)_{M A} \simeq \int_{\hat{c}}^{\oplus} n^{\prime \prime}(\sigma, \tau, \delta, \pi)(\sigma \times \tau) d \nu(\sigma, \tau)
$$

where $\nu \ll \mu_{C}$ and $n^{\prime \prime}(\sigma, \tau, \delta, \pi) \leqslant n^{\prime}(\sigma, \tau, \delta, \pi)$ for $\nu$ almost all $(\sigma, \tau)$. We may now write $n^{\prime \prime}(\sigma, \tau, \delta, \pi)=n^{\prime \prime}(\sigma, \tau, \pi)$ since, if $\pi$ is also contained in $\operatorname{Ind}_{K}^{G} \delta^{\prime}, \delta^{\prime} \in \hat{K}$, then we would have 
$\int_{\hat{C}}^{\oplus} n^{\prime \prime}(\sigma, \tau, \delta, \pi)(\sigma \times \tau) d \nu(\sigma, \tau) \simeq(\pi)_{M A} \simeq \int_{\hat{C}}^{\oplus} n_{2}^{\prime \prime}\left(\sigma, \tau, \delta^{\prime}, \pi\right)(\sigma \times \tau) d \nu_{2}(\sigma, \tau)$ and hence that $\nu \sim \nu_{2}$ and $n^{\prime \prime}=n_{2}^{\prime \prime}$ for $\nu$ almost all $(\sigma, \tau)$. If we now choose a $\mu_{C}$-measurable set $E \subseteq \hat{C}$ such that $\nu$ and $\chi_{E} \cdot \mu_{C}\left(\chi_{E}\right.$ is the characteristic function of $E$ ) have the same null sets, then by [12, p. 123] we obtain

$$
(\pi)_{M A} \simeq \int_{\hat{C}}^{\oplus} n^{\prime \prime}(\sigma, \tau, \pi)(\sigma \times \tau) d\left(\chi_{E} \cdot \mu_{C}\right)(\sigma, \tau) .
$$

If we now define

$$
n(\sigma, \tau, \pi)= \begin{cases}n^{\prime \prime}(\sigma, \tau, \pi) & \text { if }(\sigma, \tau) \in E, \\ 0 & \text { otherwise, }\end{cases}
$$

then we have $(\pi)_{M A} \simeq \int_{\hat{C}}^{\oplus} n(\sigma, \tau, \pi)(\sigma \times \tau) d \mu_{C}(\sigma, \tau)$ and the proof is complete.

Combining Theorems 8 and 15 , we now see that for $\mu_{G}$ almost all $\pi \in \hat{G}$

$$
(\pi)_{M A} \simeq \int_{\hat{C}}^{\oplus} n(\sigma, \tau, \pi)(\sigma \times \tau) d \mu_{C}(\sigma, \tau)
$$

where $n(\sigma, \tau, \pi)$ is a measurable function on $\hat{M} \times \hat{A} \times \hat{G}$. So by Anh's reciprocity theorem and Theorem 2 , we have for all $(\sigma, \tau) \in(M A)^{\wedge}$

$$
\operatorname{Ind}_{M A}^{G} \sigma \times \tau \simeq \int_{\hat{G}}^{\oplus} n(\sigma, \tau, \pi) \pi d \mu_{G}(\pi)
$$

where $n(\sigma, \tau, \pi)$ can be computed explicitly for $\pi$ in the principal series and $n(\sigma, \tau, \pi) \in\{0,1,2, \cdots, \infty\}$ for $\pi \in \hat{G}_{d^{*}}$. Note that since $\operatorname{Ind}_{M A}^{G} \sigma \times \tau$ is independent of $\tau \in \hat{A}$, we may now conclude that $n(\sigma, \tau, \pi)=n(\sigma, \pi)$.

THEOREM 16. Let $G$ be a connected semisimple real-rank one Lie group with finite center, let $\pi\left(\sigma_{1}, \tau_{1}\right), \pi\left(\sigma_{2}, \tau_{2}\right)$ be two principal series representations, let $\sigma_{1} \otimes \sigma_{2}=\Sigma_{i=1}^{n} b_{j} \sigma_{j}$, let $M_{0}$ be as in Lemma 6, and for $\chi_{1}, \chi_{2} \in$ $\operatorname{Rep}\left(M_{0}\right)$ let $I\left(\chi_{1}, \chi_{2}\right)$ denote the intertwining number for $\chi_{1}$ and $\chi_{2}$. Then $\pi\left(\sigma_{1}, \tau_{1}\right) \otimes \pi\left(\sigma_{2}, \tau_{2}\right) \simeq T_{c} \oplus T_{d}$ where $T_{c}$ is a continuous direct integral with respect to Plancherel measure on $\hat{G}$ of representations from the principal series of $G$ and $T_{d}$ is a discrete direct sum of discrete series representations. If $\operatorname{dim} g_{-2 \alpha} \neq 0$, then the multiplicity of $\pi(\sigma, \tau)$ in $T_{c}$ is either 0 or $\infty$ according to whether $\sum_{j=1}^{n} I\left((\sigma)_{M_{0}},\left(\sigma_{j}\right)_{M_{0}}\right)$ is 0 or not. If $\operatorname{dim} g_{-2 \alpha}=0$, then the multiplicity of $\pi(\sigma, \tau)$ in $T_{c}$ is finite and equals $\epsilon \cdot \Sigma_{j=1}^{n} I\left((\sigma)_{M_{0}},\left(\sigma_{j}\right)_{M_{0}}\right) b_{j}$ where $\epsilon=2$ if $\operatorname{dim} g_{\alpha}=1$ and $\epsilon=1$ if $\operatorname{dim} g_{\alpha}>1$.

Proof. All but the multiplicity of $\pi(\sigma, \tau)$ in $T_{c}$ is clear. Recall that 


$$
\pi\left(\sigma_{1}, \tau_{1}\right) \otimes \pi\left(\sigma_{2}, \tau_{2}\right) \simeq \sum_{j=1}^{n} b_{j} \operatorname{Ind}_{M A}^{G} \sigma_{j} \times \tau^{\prime}, \quad \tau^{\prime}=\tau_{1} \tau_{2},
$$

and that the multiplicity of $\pi(\sigma, \tau)$ in $\operatorname{Ind}_{M A}^{G} \sigma_{j} \times \tau^{\prime}$ equals the multiplicity of $\sigma_{j} \times \tau^{\prime}$ in $\left(\pi(\sigma, \tau)_{M A} \simeq \#(S)\left(\operatorname{Ind}_{M_{0}}^{M}(\sigma)_{M_{0}} \times{ }^{A} R\right)\right.$, viz., \#(S) (the multiplicity of $\sigma_{j}$ in $\left.\operatorname{Ind}_{M_{0}}^{M}(\sigma)_{M_{0}}\right)$.

Now let

$$
(\sigma)_{M_{0}}=\sum_{\rho \in \hat{M}_{0}} n(\sigma, \rho) \rho, \quad\left(\sigma_{j}\right)_{M_{0}}=\sum_{\rho^{\prime} \in \hat{M}_{0}} n\left(\sigma_{j}, \rho^{\prime}\right) \rho^{\prime},
$$

and

$$
\operatorname{Ind}_{M_{0}}^{M}(\sigma)_{M_{0}}=\sum_{\nu \in \hat{M}} \alpha_{\nu} \nu
$$

Then by compact reciprocity, the multiplicity of $\sigma_{j}$ in $\operatorname{Ind}_{M_{0}}^{M}(\sigma)_{M_{0}}$ is given by:

$$
\begin{aligned}
\alpha_{\sigma_{j}} & =n\left(\operatorname{Ind}_{M_{0}}^{M}(\sigma)_{M_{0}}, \sigma_{j}\right)=\sum_{\rho \in \hat{M}_{0}} n(\sigma, \rho) n\left(\operatorname{Ind}_{M_{0}}^{M} \rho, \sigma_{j}\right) \\
& =\sum_{\rho \in \hat{M}_{0}} n(\sigma, \rho) n\left(\left(\sigma_{j}\right)_{M_{0}}, \rho\right)=\sum_{\rho \in \hat{M}_{0}} n(\sigma, \rho) n\left(\sigma_{j}, \rho\right)=I\left((\sigma)_{M_{0}},\left(\sigma_{j}\right)_{M_{0}}\right) .
\end{aligned}
$$

So the multiplicity of $\pi(\sigma, \tau)$ in $\pi\left(\sigma_{1}, \tau_{1}\right) \otimes \pi\left(\sigma_{2}, \tau_{2}\right)$ will be

$$
\#(S) \cdot \sum_{j=1}^{n} I\left((\sigma)_{M_{0}},\left(\sigma_{j}\right)_{M_{0}}\right) b_{j}
$$

and the theorem is then clear from Lemma 4.

Note that even though we are not able to give the multiplicity of a discrete series representation occurring in $T_{d}$ at this time, Theorem 13 does provide us with some information about discrete series representations which do not occur in $T_{d}$.

\section{Examples.}

A. $G$ complex. When $G$ is a connected complex semisimple Lie group, we may use the techniques of $\$ 5$ to simplify some of the recent work of Floyd Williams [18]. When $G$ is complex, $M A$ is a Cartan subgroup and the principal series $\{\pi(\sigma, \tau): \sigma \in \hat{M}, \tau \in \hat{A}\}$ consitute almost all of $\hat{G}$. So by Theorems 1 and 2 of $\$ 5$ and Anh's reciprocity theorem, we know how to decompose $\pi\left(\sigma_{1}, \tau_{1}\right) \otimes \pi\left(\sigma_{2}, \tau_{2}\right) \simeq \operatorname{Ind}_{M A}^{G}\left(\sigma_{1} \sigma_{2}\right)\left(\tau_{1} \tau_{2}\right)$ once we know $(\pi(\sigma, \tau))_{M A}$ for almost all $(\sigma, \tau) \in \hat{M} \times \hat{A}$.

Let $\left\{\alpha_{1}, \cdots, \alpha_{l}\right\}$ denote the simple roots of $g,\left\{\alpha_{1}, \cdots, \alpha_{l}\right.$, $\left.\alpha_{l+1}, \cdots, \alpha_{k}\right\}$ the positive roots, $X_{j} \in 8_{-\alpha_{j}}-\{0\}$,

$$
V_{0}=\left\{\prod_{j=1}^{k} \exp z_{j} X_{j} \in V: z_{j} \in \mathrm{C}, z_{j} \neq 0 \text { for } 1 \leqslant j \leqslant l\right\} \text {, }
$$


and

$$
V^{\prime}=\left\{\prod_{j=1}^{k} \exp z_{j} X_{j} \in V: z_{j}=0,1 \leqslant j \leqslant l\right\} .
$$

Then $V-V_{0}$ has Haar measure zero and Williams shows that $V_{0} / M A$ can be identified with $V^{\prime}\left(\approx \mathrm{C}^{k-l}\right)$. Choosing $S=V^{\prime}$, we see that $S$ is either one point or infinite depending upon whether $N$ is abelian (if and only if all positive roots are simple) or not and that up to a set of Haar measure zero in $G, S$ may be identified with $M A N \backslash G / M A$. For $v \in S$, let $M_{v}=\left\{c \in M A: c^{-1} v c\right.$ $=v\}$. Then:

Lemma. $M_{v}=Z(G)$, the center of $G$, for each $v \in S$.

Proof. Clearly $Z(G) \subseteq M_{v}$ for each $v \in S$. So let $c \in M_{v}$ where $v=\exp X, X=\Sigma_{\alpha>0} X_{-\alpha}, X_{-\alpha} \in g_{-\alpha}-\{0\}$. If $\exp H=c$, then $\alpha(H)=$ 0 for all $\alpha$ [in fact $m_{X}=\{H \in h: H \cdot X=X\}=\left\{H \in h: \Sigma e^{\alpha(H)} X_{-\alpha}=\right.$ $\left.\Sigma X_{-\alpha}\right\}=\{H \in h: \alpha(H)=0$, all $\left.\alpha\}=\{0\}\right]$. Let $g=\exp \left(h_{0}+\Sigma X_{\alpha}\right)$. Then

$$
\begin{aligned}
c^{-1} g c & =\exp \left(e^{\mathrm{ad} H} h_{0}+\sum e^{\mathrm{ad} H} X_{\alpha}\right) \\
& =\exp \left(h_{0}+\sum e^{\alpha(H)} X_{\alpha}\right)=\exp \left(h_{0}+\sum X_{\alpha}\right)=g,
\end{aligned}
$$

i.e., $c \in Z(G)$. Thus by applying Mackey's subgroup theorem, we obtain

$$
(\pi(\sigma, \tau))_{M A} \simeq \int_{S}^{\oplus} \operatorname{Ind}_{M_{v}}^{M A}(\sigma \times \tau)_{M_{v}} d \mu(v) \simeq \epsilon \cdot \operatorname{Ind}_{Z(G)}^{M A}(\sigma)_{Z(G)}
$$

where $\epsilon=1$ or $\infty$ according to whether $N$ is abelian or not. Note that $(\sigma)_{Z(G)}$ is irreducible for $\sigma \in \hat{M}$. Hence we have the following:

Theorem (Williams, 1972). Let $G$ be a connected complex semisimple Lie group and $M A$ a Cartan subgroup of $G$. Suppose $\pi\left(\sigma_{1}, \tau_{2}\right), \pi\left(\sigma_{2}, \tau_{2}\right)$ are two principal series representations where $\sigma_{1}, \sigma_{2} \in \hat{M}, \tau_{1}, \tau_{2} \in \hat{A}$. Then

$$
\pi\left(\sigma_{1}, \tau_{1}\right) \otimes \pi\left(\sigma_{2}, \tau_{2}\right) \simeq \int_{\hat{G}}^{\oplus} \epsilon \cdot \pi(\sigma, \tau) d \mu_{G}(\pi)
$$

where the elements $\pi=\pi(\sigma, \tau)$ occurring in this decomposition are precisely those for which $\sigma$ and $\sigma_{1} \sigma_{2}$ coincide on $Z(G)$ and $\epsilon=1$ or $\infty$ according to whether $N$ is abelian or not.

B. $G=\operatorname{SL}(2, \mathbf{R})$. When $G=\operatorname{SL}(2, \mathbf{R})$, our techniques yield a complete solution to the problem of decomposing the tensor product of two principal series representations, since we are able to compute $(\pi)_{M A}$ for $\pi \in \hat{G}_{d}$. Recall that 


$$
\begin{aligned}
& G=\left\{\left(\begin{array}{ll}
a & b \\
c & d
\end{array}\right): a d-b c=1, a, b, c, d \in \mathbf{R}\right\}, \quad K=\operatorname{SO}(2), \\
& A=\left\{\left(\begin{array}{ll}
a & 0 \\
0 & a^{-1}
\end{array}\right): a>0\right\} \approx(0, \infty), \quad V=\left\{\left(\begin{array}{ll}
1 & 0 \\
v & 1
\end{array}\right): v \in \mathbf{R}\right\}, \\
& M=Z(G)=\left(\begin{array}{rr} 
\pm 1 & 0 \\
0 & \pm 1
\end{array}\right),
\end{aligned}
$$

$\hat{A} \approx \mathbf{R}$, and $\hat{M}=\left\{\sigma^{ \pm}\right\}$. The discrete series for $\operatorname{SL}(2, \mathbf{R})$ (see [16]) can be parameterized by nonzero half-integers and briefly described as follows: On the upper half plane $P$, for $n=1,3 / 2,2, \cdots$ (resp. $n=-1,-3 / 2,-2, \cdots)$ ), we take the Hilbert space $H_{2, n}(P)$ of holomorphic (resp. conjugate holomorphic) functions on $P$ with the inner product

$$
(f, g)_{n}=\frac{1}{\Gamma(2 n-1)} \int_{P} f(x+i y) \overline{g(x+i y)} y^{-2+2|m|} d x d y
$$

while for $n=1 / 2$ (resp. $n=-1 / 2), H_{2,1 / 2}(P)$ (resp. $H_{2,-1 / 2}(P)$ ) is the space of holomorphic (resp. conjugate holomorphic) functions on $\boldsymbol{P}$ with the property that $\int_{-\infty}^{\infty}|f(x+i y)|^{2} d x$ is bounded uniformly in $y$ for $y>0$. In this case, $f$ has boundary values almost everywhere on the real axis and if $f(x), g(x)$ denote the boundary values of $f(z), g(z)$, then the inner product is given by $\int_{-\infty}^{\infty} f(x) \overline{g(x)} d x$. For $n=\{ \pm 1 / 2, \pm 1, \pm 3 / 2, \cdots\}$, we have a representation $D_{n} \in \hat{G}$ acting on $H_{2, n}$ via

$$
\begin{aligned}
& D_{n}(g) f(z)=(b z+d)^{-2 n} f\left(\frac{a z+c}{b z+d}\right), \quad n=1 / 2,1,3 / 2, \cdots, \\
& D_{n}(g) f(z)=(b \bar{z}+d)^{-2|n|} f\left(\frac{a z+c}{b z+d}\right), \quad n=-1 / 2,-1,-3 / 2, \cdots,
\end{aligned}
$$

where $g=\left(\begin{array}{ll}a & b \\ c & d\end{array}\right)$. The discrete series of $G=\operatorname{SL}(2, \mathbf{R})$ is the family of representations $\hat{G}_{d}=\left\{D_{n}: n= \pm 1, \pm 3 / 2, \pm 2, \cdots\right\}$. The representations $D_{1 / 2}, D_{-1 / 2}$ are not square-integrable and it is known that $D_{1 / 2} \oplus D_{-1 / 2} \simeq \pi\left(\sigma^{-}, 0\right)$. For $\tau \in A \approx(0, \infty)$, let $d \mu(t)=d t / t$ denote Haar measure on $A$ and $L^{2}(A)=$ $L^{2}((0, \infty) ; d \mu)$. For $a, t \in A, f \in L^{2}(A)$, let $\rho(a) f(t)=f(a t)$ be the regular representation of $A$ and $\tilde{\rho}(a) f(t)=f\left(a^{2} t\right)$. Then

LEMMA. $\rho \simeq \tilde{\rho}$.

Proof. Let $\Psi: L^{2}(A) \rightarrow L^{2}(A)$ by $\Psi(f(t))=2^{-1 / 2} f(\sqrt{ } t), t \in(0, \infty)$. Then 


$$
\begin{aligned}
\|\Psi f\|^{2}=\int_{0}^{\infty}|\Psi f(t)|^{2} & \frac{d t}{t}=\frac{1}{2} \int_{0}^{\infty}|f(\sqrt{ } t)|^{2} \frac{d t}{t} \\
& =\frac{1}{2} \int_{0}^{\infty}|f(t)|^{2} \frac{2 t d t}{t^{2}}=\int_{0}^{\infty}|f(t)|^{2} \frac{d t}{t}=\|f\|^{2}
\end{aligned}
$$

and for $F=\Psi^{-1} f$

$$
\begin{aligned}
\left(\Psi \rho(a) \Psi^{-1} f\right)(t) & =2^{-1 / 2} \rho(a) F(\sqrt{ } t)=2^{-1 / 2} F(a \sqrt{ } t) \\
& \left.=f\left(a^{2} t\right)=\tilde{\rho}(a) f\right)(t) .
\end{aligned}
$$

LEMMA.

$$
\left(D_{n}\right)_{M A} \simeq \begin{cases}\sigma^{+} \times \rho & \text { if } n= \pm 1, \pm 2, \cdots, \\ \sigma^{-} \times \rho & \text { if } n= \pm \frac{1}{2}, \pm \frac{3}{2}, \cdots\end{cases}
$$

Proof. Let $n=1 / 2,1,3 / 2, \cdots$ and

$$
g=m a=\left(\begin{array}{rr} 
\pm 1 & 0 \\
0 & \pm 1
\end{array}\right)\left(\begin{array}{ll}
a^{-1} & 0 \\
0 & a
\end{array}\right) \in M A .
$$

As in [16], for $n>1 / 2$ we let $H_{(2, n)^{-}}(P)$ be the Hilbert space of functions on $(0, \infty)$ with the inner product

$$
(\phi, \Psi)_{n}=\frac{1}{2^{2 n-1}} \int_{0}^{\infty} \phi(t) \overline{\Psi(t)} t^{-2 n+1} d t
$$

and define the inverse Fourier-Laplace transform of a holomorphic function $f$ in $H_{2, n}(P)$ by

$$
(F f)(t)=(2 \pi)^{-1 / 2} \int_{-\infty}^{\infty} f(x+i y) e^{-i t(x+i y)} d x .
$$

This integral is independent of $y>0, F f(t)$ vanishes almost everywhere for $t<0$, and $F: H_{2, n}(P) \rightarrow H_{(2, n)^{(}(P)}$ is an isometric isomorphism.

For $n=1 / 2$, the inverse Fourier-Laplace transform on $H_{2,1 / 2}(P)$ reduces to the ordinary Fourier transform on $L_{2}(R)$, i.e.,

$$
F(f)(t)=(2 \pi)^{-1 / 2} \int_{-\infty}^{\infty} f(x) e^{-i t x} d x
$$

and, as is well-known, $F f(t)$ vanishes almost everywhere for $t<0$. Setting $H_{(2,1 / 2)}(P)=i_{2}((0, \infty), d t)$, we have that $F$ is an isometric isomorphism from $H_{2,1 / 2}(P)$ to $H_{(2,1 / 2)}(P)$. 
For $f \in H_{2, n}(P), n=1 / 2,1,3 / 2, \cdots$, and $g=m a \in M A$, we have $D_{n}(g) f(z)=(m a)^{-2 n} f\left(a^{-2} z\right)$. We now use $F$ to realize $\left(D_{n}\right)_{M A}$ on $H_{(2, n)^{-}}(P)$ : $\left(F D_{n}(g) F^{-1} g\right)(t)=\left(F D_{n}(g) f\right)(z)$ where $F^{-1} g=f$.

$$
\begin{aligned}
& =(2 \pi)^{-1 / 2} \int_{R}(m a)^{-2 n} f\left(a^{-2} z\right) e^{-i t z} d x \\
& =(2 \pi)^{-1 / 2} \int_{R}(m a)^{-2 n} f(z) e^{-i t a^{2} z} a^{2} d x \\
& =m^{-2 n} a^{2-2 n}(2 \pi)^{-1 / 2} \int_{R} f(z) e^{-i t a^{2} z} d x \\
& =m^{-2 n} a^{2-2 n} g\left(a^{2} t\right) .
\end{aligned}
$$

Set $(W(n))^{\wedge} f(t)=(2 t)^{-n+1 / 2} f(t)$. Then $(W(n))^{\wedge}$ is a unitary mapping from $H_{(2, n)^{-}}(P)$ to $H_{(2,1 / 2)^{\wedge}}(P)$ and we may now realize $\left(D_{n}\right)_{M A}$ on $H_{(2,1 / 2)^{\wedge}}(P)$ :

$$
\begin{aligned}
\left.\widehat{(W(n)} D_{n}(g) \widehat{W(n)}-1 f\right)(t) & =\widehat{W(n)} m^{-2 n} a^{2-2 n} F\left(a^{2} t\right) \\
& =(2 t)^{-n+1 / 2} m^{-2 n} a^{2-2 n} F\left(a^{2} t\right) \\
& =(2 t)^{-n+1 / 2} m^{-2 n} a^{2-2 n}\left(2 a^{2} t\right)^{n-1 / 2} f\left(a^{2} t\right) \\
& =m^{-2 n} a f\left(a^{2} t\right) .
\end{aligned}
$$

Now set $\Phi(f(t))=t^{1 / 2} f(t)$. Then $\Phi$ is a unitary mapping of $H_{(2,1 / 2)^{-}}(P)$ $=L^{2}((0, \infty), d t)$ into $L^{2}((0, \infty), d t / t)=L^{2}(A)$ and

$$
\begin{aligned}
\left(\Phi D_{n}(g) \Phi^{-1} f\right)(t) & =\left(\Phi D_{n}(g) F\right)(t)=t^{1 / 2} m^{-2 n} a F\left(a^{2} t\right) \\
& =t^{1 / 2} m^{-2 n} a a^{-1} t^{-1 / 2} f\left(a^{2} t\right)=m^{-2 n} f\left(a^{2} t\right) .
\end{aligned}
$$

For $n=1,2, \cdots$, this last representation is equivalent to $\sigma^{+} \times \rho$ while for $n=1 / 2,3 / 2, \cdots$, this last representation is equivalent to $\sigma^{-} \times \rho$. A similar argument holds for $n=-1 / 2,-1,-3 / 2, \cdots$ and so the lemma is now clear.

Now recall that $M A$ acts on $V$ in two nonzero orbits and so $(\pi(\sigma, \tau))_{M A}$ $\simeq 2 \operatorname{Ind}_{M}^{M A} \sigma \simeq 2(\sigma \times \rho)$ for $\sigma \in \hat{M}, \tau \in \hat{A}$. Thus we have

TheOREM (PuKANSZKY, 1960). Let $\pi\left(\sigma_{1}, \tau_{1}\right), \pi\left(\sigma_{2}, \sigma_{2}\right), \sigma_{1}, \sigma_{2} \in$ $\hat{M}=\left\{\sigma^{ \pm}\right\}, \tau_{1}, \tau_{2} \in \hat{A}$ be two representations from the principal series of $G=\operatorname{SL}(2, \mathbf{R})$. Then 


$$
\pi\left(\sigma_{1}, \tau_{1}\right) \otimes \pi\left(\sigma_{2}, \tau_{2}\right) \simeq \begin{cases}2 \int_{\hat{G}}^{\oplus} \pi\left(\sigma^{+}, \tau\right) d \mu_{G}(\pi) \oplus \sum_{n= \pm 1, \pm 2, \ldots} \oplus D_{n} \\ 2 \int_{\hat{G}}^{\oplus} \pi\left(\sigma^{-}, \tau\right) d \mu_{G}(\pi) \oplus \sum_{n= \pm 3 / 2, \ldots} \sigma_{n} \sigma_{1} D_{2}=\sigma^{+}, \\ \text {if } \sigma_{1} \sigma_{2}=\sigma^{-} .\end{cases}
$$

C. $G \approx \mathrm{SO}_{e}(3,1) \approx \mathrm{SL}(2, \mathrm{C}) /\{ \pm e\}$. When $G=\mathrm{SO}_{e}(3,1), S$ is one point, $M_{0}=\{e\},(\pi(\sigma, \tau))_{M A} \simeq \operatorname{Ind}_{\{e\}}^{M A} 1 \simeq{ }^{M A} R$ for all $(\sigma, \tau) \in \hat{M} \times \hat{A}$, and there is no discrete series for $G$. Thus we obtain:

THEOREM (MACKEY, 1952-NAǏMARK, 1958). Let $\pi\left(\sigma_{1}, \tau_{1}\right), \pi\left(\sigma_{2}, \tau_{2}\right)$ where $\sigma_{1}, \sigma_{2} \in \hat{M}=\mathrm{SO}(2)^{\wedge}, \tau_{1}, \tau_{2} \in \hat{A}$, be two representations from the principal series of $G=\mathrm{SO}_{e}(3,1)$. Then

$$
\pi\left(\sigma_{1}, \tau_{1}\right) \otimes \pi\left(\sigma_{2}, \tau_{2}\right) \simeq \int_{\hat{G}}^{\oplus} \pi(\sigma, \tau) d \mu_{\hat{G}}(\pi) .
$$

Note that Theorem 16 also yields a complete solution to the decomposition of the tensor product of two principal series representations for $G=$ $\mathrm{SO}_{e}(2 n+1,1), n \geqslant 1$, since in this case $\hat{G}_{d}=\phi$.

D. $G=\operatorname{SL}(n, R), n \geqslant 3$. We shall now show how the techniques developed in $\$ 5$ yield a complete solution to the problem of decomposing the tensor product of two (minimal) principal series representations of $G=\operatorname{SL}(n, \mathbf{R})$, $n \geqslant 3$. Let $D\left(a_{1}, \cdots, a_{n}\right)$ denote the diagonal matrix in $G$ with entries $a_{1}, \cdots, a_{n}$ and let $e_{i}\left(D\left(a_{1}, \cdots, a_{n}\right)\right)=a_{i}, i=1, \cdots, n$. We have that $\boldsymbol{8}=\{X \in \mathrm{gl}(n, \mathrm{R}): \operatorname{tr}(X)=0\}, \quad \mathfrak{l}=\left\{X \in \mathbf{8}: X^{t}=-X\right\}$, $\mathfrak{p}=\left\{X \in \mathfrak{g}: X^{t}=X\right\}, \quad a=\left\{D\left(a_{1}, \cdots, a_{n}\right): a_{i} \in \mathbf{R}, \sum_{i=1}^{n} a_{i}=0\right\}$, $\theta$ is negative transpose, $\Delta=\left\{ \pm\left(e_{i}-e_{j}\right): 1 \leqslant i \leqslant j \leqslant n\right\}, \Delta^{+}=\left\{e_{i}-e_{j}\right.$ : $1 \leqslant i \leqslant j \leqslant n\}, \quad \boldsymbol{g}_{e_{i}-e_{j}}=\mathbf{R} \cdot E_{i j}\left(E_{i j}\right.$ is the $n \times n$ matrix consisting of all zeros and a single one in the $i j$ th place), the simple roots are $\left\{e_{i}-e_{2}, e_{2}-e_{3}\right.$, $\left.\cdots, e_{n-1}-e_{n}\right\}, K=\mathrm{SO}(n), A$ is the subgroup of all diagonal matrices with positive diagonal elements, $N$ is the subgroup of all matrices which are one on the diagonal and zero below and $M$ is the subgroup of all matrices which are \pm 1 on the diagonal and zero elsewhere. For $n$ odd, the center of $G$ is $Z(G)$ $=\{e\}$ while for $n$ even, $Z(G)=\{ \pm e\}$. For $n$ even, we put $(Z(G))^{\wedge}=$ $\left\{\chi^{+}, \chi^{-}\right\}$. The group of all diagonal matrices in $G, M A$, is a Cartan subgroup of $G$ and the principal series of $G$ is the family of representations $\left\{\pi_{0}(\sigma, \tau)\right.$ $\left.=\operatorname{Ind}_{M A N}^{G} \sigma \times \tau: \quad \sigma \in \hat{M}, \tau \in \hat{A}\right\}$.

For $n \geqslant 3$, Rank $G \neq \operatorname{Rank} K$ and so there is no discrete series to contend with. However, $G$ has Cartan subgroups not conjugate to $M A$ and for 
each conjugacy class there is a cuspidal parabolic subgroup and a corresponding family of continuous series representations, $F$, for which $\mu_{G}(F) \neq 0$. Let $P_{0}=M A N$, a minimal parabolic subgroup of $G$. We now construct a complete set of nonassociate cuspidal parabolic subgroups of $G$. If $t$ is the largest integer such that $2 t \leqslant n$, then there are exactly $t$ mutually nonconjugate Cartan subgroups which are not conjugate to $M A[17$, vol. I, p. 95] and so there are $t+1$ nonassociate cuspidal parabolic subgroups for $G$. The following construction is detailed in [17, vol. I, pp. 66-78]. Let $\theta_{1}=\left\{e_{1}-e_{2}\right\}, \theta_{2}=$ $\left\{e_{1}-e_{2}, e_{3}-e_{4}\right\}, \cdots, \theta_{t}=\left\{e_{1}-e_{2}, e_{3}-e_{4}, \cdots, e_{2 t-1}-e_{2 t}\right\}$. Let $\left\langle\theta_{i}\right\rangle$ denote the set of those $\lambda \in \Delta$ which are linear combinations of the elements in $\theta_{1},\left\langle\theta_{i}\right\rangle^{ \pm}=\Delta^{ \pm} \cap\left\langle\theta_{i}\right\rangle$, and $\mathfrak{n}^{ \pm}\left(\theta_{i}\right)=\Sigma_{\lambda \in\left\langle\theta_{i}\right\rangle^{ \pm}} \mathbb{g}_{\lambda}$ (note that $\left\langle\theta_{i}\right\rangle^{ \pm}$ $=\theta_{i}$ ). If $\mathbf{g}\left(\theta_{i}\right)$ denotes the subalgebra of $\boldsymbol{g}$ generated by $\mathfrak{n}^{+}\left(\theta_{i}\right)+\mathfrak{n}^{-}\left(\theta_{i}\right)$, then $\mathfrak{g}\left(\theta_{i}\right)=\mathfrak{l}\left(\theta_{i}\right)+\mathfrak{p}\left(\theta_{i}\right)$ where $\mathfrak{t}\left(\theta_{i}\right)=\mathfrak{g}\left(\theta_{i}\right) \cap \mathfrak{l}, \mathfrak{p}\left(\theta_{i}\right)=\mathfrak{g}\left(\theta_{i}\right) \cap \mathfrak{p}$. Furthermore, $g\left(\theta_{i}\right)$ is semisimple and $g\left(\theta_{i}\right)=\mathfrak{f}\left(\theta_{i}\right)+\mathfrak{p}\left(\theta_{i}\right)$ is a Cartan decomposition. For $\lambda \in \theta_{i}$ we fix $X \in \mathbb{8}_{\lambda}$ with $-B(X, \theta X)=1$. Then $[\theta X, X]=Q_{\lambda} \in g\left(\theta_{i}\right)$. Let $a_{i}$ denote the orthogonal complement (relative to $B_{\theta}$ ) of $\Sigma_{\lambda \in \theta_{i}} \mathbf{R} \cdot Q_{\lambda}$ in a. For $x=x E_{12} \in 8_{e_{1}-e_{2}}, Q_{\lambda}=$ $D\left(+x^{2},-x^{2}, 0, \cdots, 0\right)$ and $\operatorname{tr}\left(D\left(a_{1}, \cdots, a_{n}\right) Q_{\lambda}\right)=x^{2}\left(a_{1}-a_{2}\right)$. Since $B(X, Y)$ is a multiple of $\operatorname{tr}(X Y)$, we have that $a_{1}=D\left(a_{1}, a_{1}, a_{3}, \cdots, a_{n}\right)$. Similarly, we have for $k \leqslant t, a_{k}=D\left(a_{1}, a_{1}, a_{2}, a_{2}, \cdots, a_{k}, a_{k}, a_{2 k+1}, \cdots, a_{n}\right)$. Now set $\mathfrak{n}_{i}=\Sigma_{\lambda \in \Delta^{+}-\theta_{i}} g_{\lambda}, \mathfrak{v}_{i}=\theta\left(\mathfrak{n}_{i}\right)$, and

$$
\mathfrak{p}_{i}=\mathfrak{p}_{0}+\mathfrak{n}^{-}\left(\theta_{i}\right)=\mathfrak{n}^{+}\left(\theta_{i}\right)+\mathfrak{n}^{-}\left(\theta_{i}\right)+\sum_{\lambda \in \theta_{i}} \mathrm{R} Q_{\lambda}+\mathfrak{a}_{i}+\mathfrak{n}_{i}
$$

$\left(\mathfrak{p}_{0}=L A\left(P_{0}\right)\right)$. Denote by $A_{i}, N_{i}, V_{i}$ the analytic subgroups of $G$ corresponding respectively to $\mathfrak{a}_{i}, \mathfrak{n}_{i}, \mathfrak{b}_{i}$. Note that $V_{i}$ has the following form

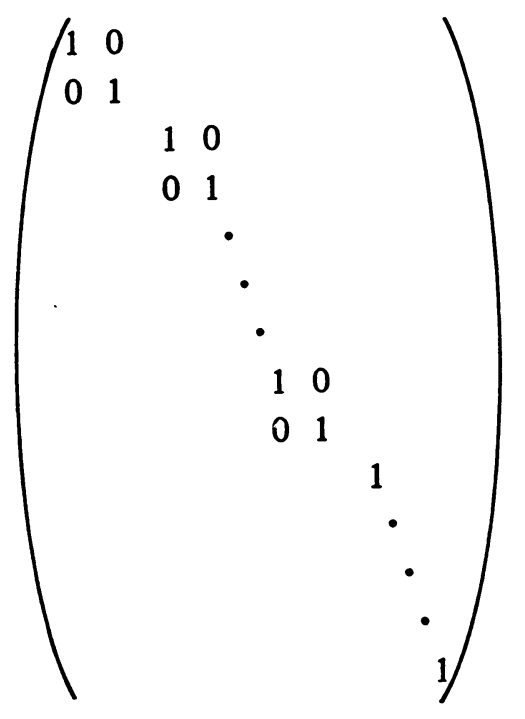


where there are $i$ blocks of the form $\left(\begin{array}{ll}1 & 0 \\ 0 & 1\end{array}\right)$. Let

i.e.,

$$
\mathfrak{m}_{i}=\mathfrak{n}^{+}\left(\theta_{i}\right)+\mathfrak{n}^{-}\left(\theta_{i}\right)+\sum_{\lambda \in \theta_{i}} \mathbf{R} Q_{\lambda}
$$

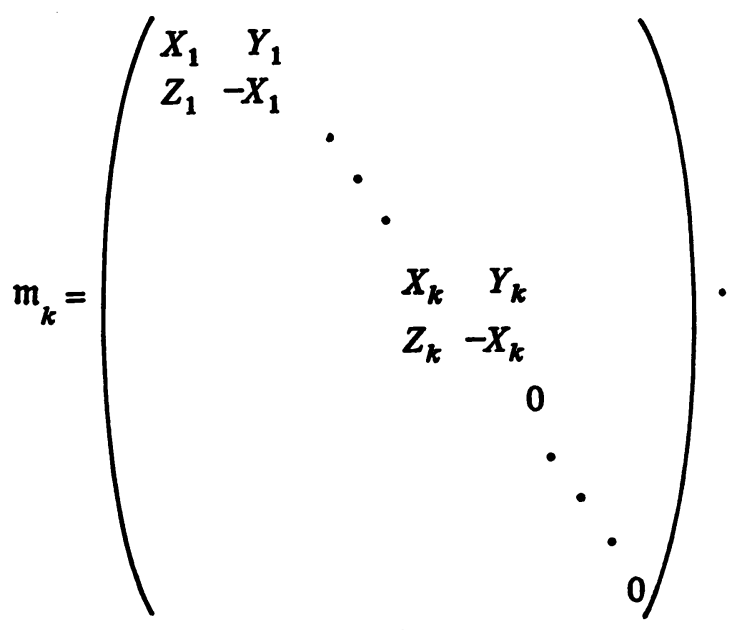

Let $M_{\theta_{i}}$ denote the analytic subgroup of $G$ with Lie algebra $m_{i}$ and $M_{\theta_{i}}(K)$ denote the centralizer of $a_{i}$ in $K$. Then

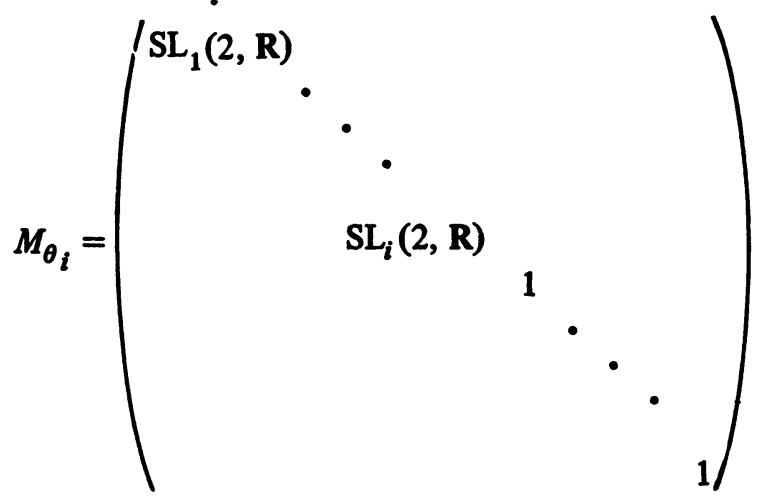

[the indices indicate copies of $\operatorname{SL}(2, R)$ ] while $M_{\theta_{i}}(K)$ has the form

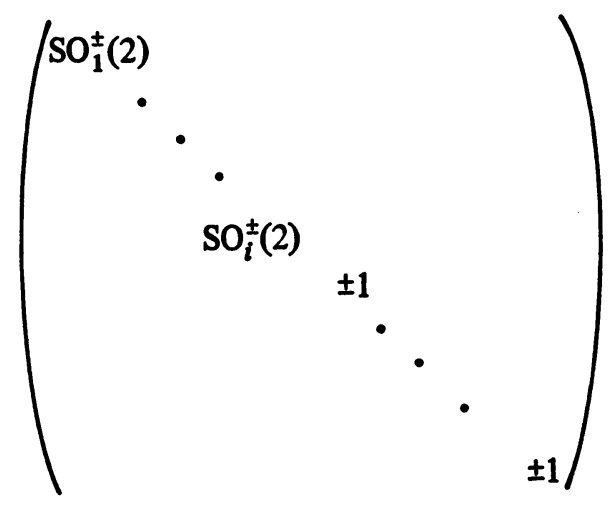


where the determinant must, of course, be 1. Finally, let $M_{i}=M_{\theta_{i}}=$ $M_{\theta_{i}}(K) M_{\theta_{i}}$ Then
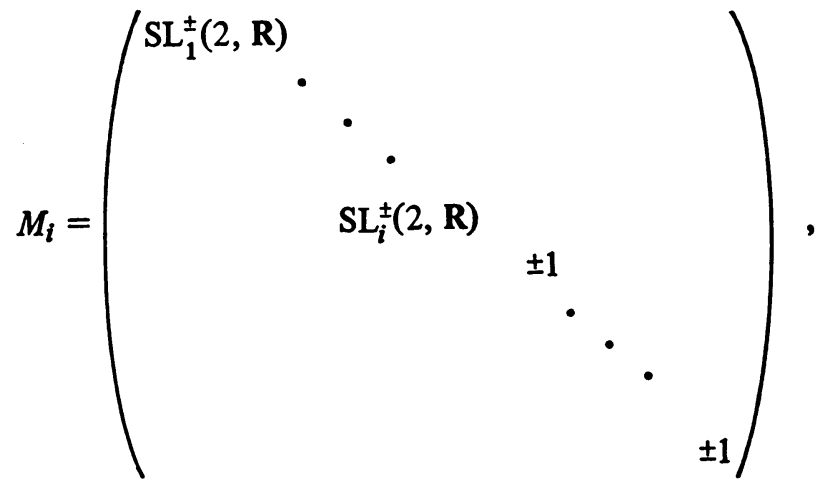

$P_{i}=M_{i} A_{i} N_{i}$ is a parabolic subgroup of $G$ containing $P_{0}$ (with Langlands decomposition $\left.M_{i} A_{i} N_{i}\right)$, and $P_{i} V_{i}$ is a dense open submanifold of $G$ whose complement has zero Haar measure. Clearly each $P_{i}$ for $i=0,1, \cdots, t$ is cuspidal and since $\operatorname{dim} A_{i} \neq A_{j}, i \neq j$, they are all nonassociate. Thus $P_{0}$, $\cdots, P_{t}$ is a complete set of nonassociate cuspidal parabolic subgroups of $G$. We shall denote the continuous series corresponding to $P_{i}$ by $\left\{\pi_{i}(\sigma, \tau)=\right.$ $\left.\operatorname{Ind}_{P_{i}}^{G} \sigma \times \tau: \quad \sigma \in \hat{M}_{i_{d}}, \tau \in \hat{A}_{i}\right\}$.

Theorem 1 now says $\pi_{0}\left(\dot{\sigma}_{1}, \tau_{1}\right) \otimes \pi_{0}\left(\sigma_{2}, \tau_{2}\right) \simeq \operatorname{Ind}_{M A}^{G}\left(\sigma_{1} \sigma_{2} \times \tau_{1} \tau_{2}\right)$. Thus, by Theorem 2 and Anh's reciprocity formula, we need only find the multiplicity of $\sigma_{1} \sigma_{2} \times \tau_{1} \tau_{2}$ in $(\pi)_{M A}$ for $\mu_{G}$-almost all $\pi \in \hat{G}$, i.e., for almost all $\pi_{i}(\sigma, \tau), i=0, \cdots, t$. Since the various continuous series representations are all induced representations, we may apply the subgroup theorem.

(1) Let $\pi_{0}(\sigma, \tau)$ be a principal series representation. Since the action of $M A$ on $M A N \backslash G$ on the left corresponds to the action of $M A$ on $V$ by inner automorphism, we may identify $M A N \backslash G / M A$ with $V / M A$. Let $\mathfrak{v}=L A(V)$ and $\mathfrak{v}^{0}=\left\{\Sigma_{\alpha \in \Delta^{-}} X_{\alpha}: X_{\alpha} \neq 0\right.$ for each $\left.\alpha \in \Delta^{-}\right\}$. Then $\mathfrak{b}^{0}$ is an open, dense, conull subset of $\mathfrak{v}$ invariant under the action of $M A$. Let $V^{0}=\exp \mathfrak{v}^{0}$. Then $V^{0}$ is a dense, conull subset of $V$ and the subgroups $M A N, M A$ will be regularly related in $G$ if we can show $V^{0} / M A$ is countably separated. Since $V^{0} / M A \approx \mathfrak{b}^{0} / M A$ (recall that exp is a diffeomorphism and takes orbits to orbits), it suffices to show ${ }^{0} / M A$ is countably separated. This will follow from $[3, \mathrm{p} .42]$ once we show that the orbits in $0^{0} / M A$ are locally closed [a subset $Z$ of a topological space $Y$ is locally closed if $Z$ is the intersection of an open and closed set]. Since $M$ is compact, it suffices to show that the orbits in $\mathfrak{v}^{0} / A$ are locally closed.

Now let $X=\Sigma X_{\alpha} \in \mathfrak{v}^{0}, Y=\Sigma Y_{\alpha} \in \mathfrak{v}^{0}$, and $\left\{\exp H_{n}\right\}$ be a sequence in $A$ with $\left(\exp H_{n}\right) \cdot X \rightarrow Y$. Then 


$$
\begin{aligned}
\left(\exp H_{n}\right) \cdot X & =\sum e^{\alpha\left(H_{n}\right)} X_{\alpha} \rightarrow \sum Y_{\alpha} \\
& \Rightarrow e^{\alpha\left(H_{n}\right)} \rightarrow Y_{\alpha} / X_{\alpha}>0 \text { for each } \alpha \in \Delta^{-} \\
& \Rightarrow \alpha\left(H_{n}\right) \text { converges for each } \alpha \in \Delta^{-} \\
& \Rightarrow \beta\left(H_{n}\right) \text { converges for each } \beta \in a^{*} \\
& \Rightarrow \exists H \in a \text { such that } \beta\left(H_{n}\right) \rightarrow \beta(H) \text { for all } \beta \in a^{*} .
\end{aligned}
$$

Now $\exp H \in A$ and

$$
\left(\exp H_{n}\right) \cdot X=\sum_{\alpha \in \Delta^{-}} e^{\alpha\left(H_{n}\right)} X_{\alpha} \rightarrow \sum_{\alpha \in \Delta^{-}} e^{\alpha\left(H_{n}\right)} X_{\alpha}=(\exp H) \cdot X .
$$

Thus

$$
(\exp H) \cdot X=Y, \quad Y \in O_{X}=\{a \cdot X: a \in A\},
$$

and $O_{X}$ is closed in $v^{0}$.

We now show that the stability subgroup of any $X \in \mathfrak{v}^{0}$ under the action of $M A$ equals $Z(G)$. Let $c=D\left(c_{1}, \cdots, c_{n}\right) \in M A$ and let

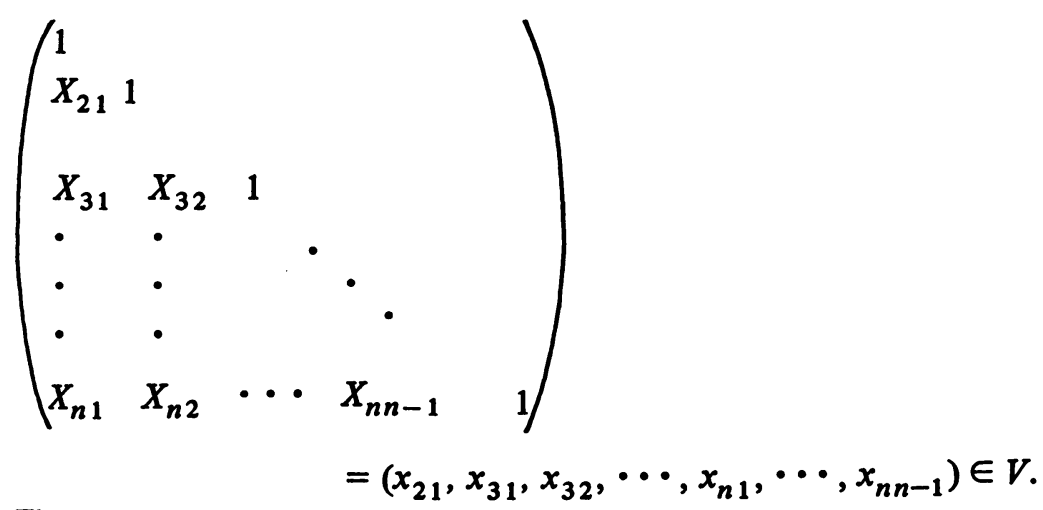

Then

$$
\begin{gathered}
c \cdot\left(x_{21}, \cdots, x_{i j}, \cdots, x_{n n-1}\right)=c\left(x_{21}, \cdots, x_{i j}, \cdots, x_{n n-1}\right) c^{-1} \\
=\left(c_{2} c_{1}^{-1} x_{21}, \cdots, c_{i} c_{j}^{-1} x_{i j}, \cdots, c_{n} c_{n-1}^{-1} x_{n n-1}\right) .
\end{gathered}
$$

Now let $x=x E_{i j} \in g_{e_{j}-e_{i}}, j<i$. Then $\exp X=I+x E_{i j}$, and $\exp \left(c_{i} c_{j}^{-1} X\right)$ $=c \cdot \exp (X)=\exp \operatorname{Ad}(c) X=\exp (c \cdot X)$. Thus $c \cdot X=c_{i} c_{j}^{-1} x E_{i j}$ and $M A$ acts in one nonzero orbit on each root space. Now suppose $X=\Sigma_{j<i} x_{i j} E_{i j} \in$ $\vee^{0}$ and $c=D\left(c_{1}, \cdots, c_{n}\right) \in M A$ with $c \cdot X=X$. Then $c \cdot X=$ $\Sigma_{j<i} c_{i} c_{j}^{-1} x_{i j} E_{i j}=\Sigma x_{i j} E_{i j}$ and $x_{i j} \neq 0, j<i$, implies that $c_{n-1} c_{n-2}^{-1}=$ $c_{n} c_{1}^{-1}=\cdots=c_{n} c_{n-2}^{-1}=1$, i.e., $c_{n}=c_{1}=c_{2}=\cdots=c_{n-2}=c_{n-1}$.

Since $1=\Pi_{i=1}^{n} c_{i}=c_{n}^{n}$, we have that $c \in Z(G)$. Thus 
$\left\{c \in M A: c \cdot X=X\right.$ for $\left.X \in \mathfrak{v}^{0}\right\}$

$$
=\left\{c \in M A: c \cdot v=v \text { for } v \in V^{0}=\exp v^{0}\right\}=Z(G) \text {. }
$$

Now by Mackey's subgroup theorem, we have

$$
\begin{aligned}
\left(\operatorname{Ind}_{M A N}^{G} \sigma \times \tau\right)_{M A} & \simeq \int_{V^{0} / M A}^{\oplus} \operatorname{Ind}_{Z(G)}^{M A}(\sigma)_{Z(G)} d \nu(v) \\
& \simeq \infty\left(\operatorname{Ind}_{Z(G)}^{M}(\sigma)_{Z(G)} \times \rho\right)
\end{aligned}
$$

where $\rho$ denotes the regular representation of $A$ and $\nu$ is any admissible measure on $V / M A$.

(2) We now find $\left(\operatorname{Ind}_{P_{i}}^{G} \sigma \times \tau\right)_{M A}$ for $i=1, \cdots, t$. Since the action of $M A$ on $P_{i} \backslash G$ corresponds to the action of $M A$ on $V_{i}$ by inner automorphism, we may identify $P_{i} \backslash G / M A$ with $V_{i} / M A$. Let $V_{i}^{0}=V_{i} \cap V^{0}$. Then $V_{i}^{0}$ is a dense, conull subset of $V_{i}$ such that the stability subgroup at any $v \in$ $V_{i}^{0}$ is equal to $Z(G)$ (same proof as in (1)). By an argument similar to that in (1), we have that $V_{i}^{0} / M A$ is countably separated. If we now let $v_{i}$ denote any admissible measure on $V_{i} / M A$, we have by Mackey's subgroup theorem

$$
\left(\operatorname{Ind}_{P_{i}}^{G} \sigma \times \tau\right)_{M A} \simeq \int_{V_{i} / M A}^{\oplus} \operatorname{Ind}_{Z(G)}^{M A}(\sigma)_{Z(G)} d \nu_{i}(v)
$$

For

$$
n=3, \quad t=1, \quad V_{1}=\left(\begin{array}{ccc}
1 & 0 & 0 \\
0 & 1 & 0 \\
* & * & 1
\end{array}\right), Z(G)=\{e\}
$$

there is one nonzero orbit in $V_{1}^{0} / M A, M_{1}=\mathrm{SL}^{ \pm}(2, \mathrm{R})$, and for $\sigma \in \hat{M}_{1_{d}}$ we have $(\sigma)_{Z(G)}=\infty \cdot 1$. Thus

$$
\left(\operatorname{Ind}_{P_{1}}^{G} \sigma \times \tau\right)_{M A} \simeq \infty \operatorname{Ind}_{\{e\}}^{M A} 1 \simeq \infty{ }^{M A} R .
$$

For $n>3, \operatorname{dim}\left(V_{i} / M A\right) \geqslant 1$ and so for $i=1, \cdots, t$

$$
\left(\operatorname{Ind}_{P_{i}}^{G} \sigma \times \tau\right)_{M A} \simeq \infty\left(\operatorname{Ind}_{Z(G)}^{M}(\sigma)_{Z(G)} \times \rho\right) .
$$

So for $n$ odd and $i=0,1, \cdots, t$ we have $\left(\operatorname{Ind}_{P_{i}}^{G} \sigma \times \tau\right)_{M A} \simeq{ }^{M A} R$. For $n$ even, we let

$$
T^{+}=\sum_{\sigma \in \hat{M},(\sigma)_{Z(G)}=\mathrm{x}^{+}} \sigma \quad \text { and } \quad T^{-}=\sum_{\sigma \in \hat{M},(\sigma)_{Z(G)}=\mathrm{x}^{-}} \sigma .
$$

Then for $i=0,1, \cdots, t$

$$
\left(\operatorname{Ind}_{P_{i}}^{G} \sigma \times \tau\right)_{M A} \simeq \begin{cases}\infty\left(T^{+} \times \rho\right) & \text { if }(\sigma)_{Z(G)} \simeq \infty \chi^{+}, \\ \infty\left(T^{-} \times \rho\right) & \text { if }(\sigma)_{Z(G)} \simeq \infty \chi^{-} .\end{cases}
$$

The following theorem is now clear. 
THEOREM. Let $\pi_{0}\left(\sigma_{1}, \tau_{1}\right), \pi_{0}\left(\sigma_{2}, \tau_{2}\right)$ be two principal series representations for $G=\operatorname{SL}(n, \mathbf{R})$ for $n \geqslant 3$. Then if $n$ is odd, $\pi_{0}\left(\sigma_{1}, \tau_{1}\right) \otimes \pi_{0}\left(\sigma_{2}, \tau_{2}\right)$ $\simeq{ }^{G} R$, while if $n$ is even

$$
\pi_{0}\left(\sigma_{1}, \tau_{1}\right) \otimes \pi_{0}\left(\sigma_{2}, \tau_{2}\right) \simeq \begin{cases}\int_{\hat{G}_{+}}^{\oplus} \infty \pi d \mu_{G}(\pi) & \text { if }\left(\sigma_{1} \sigma_{2}\right)_{Z(G)}=\chi^{+}, \\ \int_{\hat{G}_{-}}^{\oplus} \infty \pi d \mu_{G}(\pi) & \text { if }\left(\sigma_{1} \sigma_{2}\right)_{Z(G)}=\chi^{-},\end{cases}
$$

where $\hat{G}_{+}=\left\{\pi \in \hat{G}:(\pi)_{Z(G)}=\infty \chi^{+}\right\}, \hat{G}_{-}=\left\{\pi \in \hat{G}:(\pi)_{Z(G)}=\infty \chi^{-}\right\}$.

\section{REFERENCES}

1. N. Anh, Restriction of the principal series of $\mathrm{SL}(n, \mathrm{C})$ to some reductive subgroups, Pacific J. Math. 38 (1971), 295-313.

2. C. Chevalley, Theory of Lie groups. Vol. I, Princeton Math. Ser., vol. 8, Princeton Univ. Press, Princeton, N. J., 1946. MR 7, 412.

3. E. G. Effros, Transformation groups and $C^{*}$-algebras, Ann. of Math. (2) 81 (1965), 38-55. MR 30 \#5175.

4. J. Ernest, A decomposition theory for unitary representations of locally compact groups, Trans. Amer. Math. Soc. 104 (1962), 252-277. MR 25 \#3383.

5. S. Helgason, Differential geometry and symmetric spaces, Pure and Appl. Math., vol. 12, Academic Press, New York, 1962. MR 26 \#2986.

6. K. Johnson, Composition series and intertwining operators for the spherical principal series. II (to appear).

7. A. Kleppner and R. L. Lipsman, The Plancherel formula for group extensions, Ann. Sci. Ecole Norm. Sup. 5 (1972), 459-516.

8. A. W. Knapp and E. M. Stein, Intertwining operators for semi-simple groups, Ann. of Math. (2) 93 (1971), 489-578.

9. B. Kostant, On the existence and irreducibility of certain series of representations, Bull. Amer. Math. Soc. 75 (1969), 627-642. MR 39 \#7031.

10. R. A. Kunze and E. M. Stein, Uniformly bounded representations. III: Intertwining operators for the principal series on semi-simple groups, Amer. J. Math. 89 (1967), 385-442. MR 38 \#269.

11. R. L. Lipsman, On the characters and equivalence of continuous series representations, J. Math. Soc. Japan 23 (1971), 452-480. MR 44 \#6906.

12. G. Mackey, Induced representations of locally compact groups. I, Ann. of Math. (2) 55 (1952), 101-139. MR 13, 434.

13. - Induced representations of locally compact groups. II. The Frobenius reciprocity theorem, Ann. of Math. (2) 58 (1953), 193-221. MR 15, 101.

14. M. A. Naimark, Decomposition of the tensor product of irreducible representations of the proper Lorentz group into irreducible representations, Trudy Moskov. Mat. Obšč. 8 (1959), 121-153; English transl., Amer. Math. Soc. Transl. (2) 36 (1964), 101-136. MR 22 \#4966.

15. L. Pukánszky, On the Kronecker products of irreducible representations of the $2 \times 2$ real unimodular group. I, Trans. Amer. Math. Soc. 100 (1961), 116-152. MR 30 \#3177. 
16. P. J. Sally, Jr., Analytic continuation of the irreducible unitary representations of the universal covering group of SL $(2, R)$, Mem. Amer. Math. Soc. No. 69 (1967). MR 38 \#3380.

17. G. Warner, Harmonic analysis on semi-simple Lie groups. Vols. 1, 2, Die Grundlehren der math. Wissenschaften, Bände 188, 189, Springer-Verlag, Berlin and New York, 1972.

18. F. Williams, Tensor products of principal series representations, Lecture Notes in Math., vol. 358, Springer-Verlag, Berlin and New York, 1973.

DEPARTMENT OF MATHEMATICS, UNIVERSITY OF PENNSYLVANIA, PHILADELPHIA, PENNSYLVANIA 19104 